\title{
CONSISTENCY RESULTS CONCERNING SUPERCOMPACTNESS
}

\author{
BY
}

\author{
TELIS K. MENAS( ${ }^{1}$ )
}

\begin{abstract}
A general framework for proving relative consistency results with regard to supercompactness is developed. Within this framework we prove the relative consistency of the assertion that every set is ordinal definable with the statement asserting the existence of a supercompact cardinal. We also generalize Easton's theorem; the new element in our result is that our forcing conditions preserve supercompactness.
\end{abstract}

Introduction. The framework for our results is "backward Easton forcing": forcing conditions are constructed in the ground model by an iteration similar to the iteration described in the Solovay-Tennenbaum paper [12], the essential difference being that at the limit stages of the construction one takes the inverse limit (instead of the direct limit) of the conditions constructed at the previous stages. Backward Easton forcing is independent from large cardinal theory. Indeed, large cardinals are mentioned only in the latter part of this paper.

The concept of supercompactness is due to Solovay [7]. We shall need only the most elementary facts concerning supercompact cardinals, which we provide in $\$ 0$.

The essential idea of the backward Easton forcing constructions is probably due to R. Jensen [unpublished, 1965]. A few years later and independently of Jensen's work, F. Tall used similar constructions to obtain the consistency of various conjectures in topology [14]. J. Silver realized the importance of these methods to the theory of large cardinals, and he refined and extended them to a method, to which we refer as the "Silver forcing method", for preserving certain large cardinal properties in suitable Cohen extensions. By this method Silver

Received by the editors September 9, 1974.

AMS (MOS) subject classifications (1970). Primary $02 \mathrm{KO5}, 02 \mathrm{~K} 35$.

Key words and phrases. Large cardinals, backward Easton forcing, ordinal definability, supercompactness.

(1) This paper is essentially Chapter 4 of the author's thesis titled On strong compactness and supercompactness written under the supervision of Dr. Robert Solovay to whom the author is grateful. Parts of this work were supported by an NSF Fellowship and by the NSF Grant GP-33951. 
obtained the consistency of the failure of the G. C. H. at a measurable cardinal [11].

The essential ideas of backward Easton forcing are incorporated in conditions II and IV of our notion of a very fine system and in Proposition 11 and Corollary 12. Silver's ideas are incorporated in condition III of a very fine system, in the generality of Proposition 11 and its corollary, and in a technique employed in the lemma of Theorem 18.

In $\S 0$ of this paper we record a few of our conventions (the rest are dealt with as they are needed) and state some facts about supercompact cardinals.

$\S 1$ is devoted to an abstract development of the Silver forcing method which incorporates and extends the key ideas stated by Silver in a brief mimeographed account distributed in the summer of 1971.

$\$ 2$ contains the two main theorems stated above and related results.

0 . ZF is Zermelo-Fraenkel set theory and ZFC is ZF plus the axiom of choice. We shall consider only standard models of ZFC. Let $M$ be a model of ZFC and $\tau\left(v_{0}, \ldots, v_{n}\right)$ a "term" of ZFC. Define $\varphi\left(v_{0}, \ldots, v_{n} v_{n+1}\right)$ to be the formula " $\tau\left(v_{0}, \ldots, v_{n}\right)=v_{n+1}$ " of ZF. Then for $x_{0}, \ldots, x_{n}, x_{n+1}$ in $M$, we say that "in $M, \tau\left(x_{0}, \ldots, x_{n}\right)=x_{n+1}$ " or " $M \vDash \tau\left(x_{0}, \ldots, x_{n}\right)=x_{n+1}$ " to mean that $M \vDash \varphi\left(x_{0}, \ldots, x_{n} x_{n+1}\right)$. Sometimes when we are not working in $M$, we write " $x_{n+1}=\tau^{M}\left(x_{0}, \ldots, x_{n}\right)$ " for " $M \vDash \tau\left(x_{0}, \ldots, x_{n}\right)=x_{n+1}$ ".

If $A$ and $B$ are sets, ${ }^{A} B$ is the set of all functions with domain $A$ and range a subset of $B$. For $f \in{ }^{A} B$ and $W \subseteq A, f[W]=\{x:(\exists y \in W)(f(y)=x)\} . p(A)$ is the set of all subsets of $A$ unless otherwise stated. If $\lambda$ is a cardinal, we say that $A$ is closed under $\lambda$-sequences if every function from $\lambda$ into $A$ is in $A$.

Small Greek letters almost always denote ordinals. Exceptions are clearly stated. Cardinals are initial or finite ordinals and are usually denoted by the letters " $\kappa$ ", " $\nu$ " and " $\lambda$ ". If $A$ is a set, $|A|$ is the cardinality of $A$. The term "cardinal" is generally reserved for infinite cardinals. If $\kappa$ and $\lambda$ are cardinals, $p_{\kappa} \lambda$ is the set of all subsets of $\lambda$ of cardinality less than $\kappa, \kappa^{\lambda}=\left.\right|^{\lambda} \kappa \mid$, and $\lambda^{r}=$ $\|\left\{{ }^{\alpha} \lambda: \alpha<\kappa\right\} \mid$. We reserve the term "inaccessible" for strongly inaccessible cardinals.

A two-valued measure $\mu: p(X) \rightarrow 2$ on a nonempty set $X$ is $\kappa$-additive if for every $\alpha<\kappa$ and $f: \alpha \rightarrow p(X)$ so that $\mu(f(\beta))=1$ for every $\beta<\alpha, \mu\left(\bigcap_{\beta<\alpha} f(\beta)\right)$ $=1$.

Let $\mu$ be a two-valued measure on $p_{\kappa} \lambda$. $\mu$ is normal if

(i) $\mu$ is $\kappa$-additive.

(ii) For all $\alpha<\lambda, \mu\left(\left\{x \in p_{k} \lambda: \alpha \in x\right\}\right)=1$.

(iii) For every function $f$ from $p_{\kappa} \lambda$ into $\lambda$, if

$$
\mu\left(\left\{x \in p_{\kappa} \lambda: f(x) \in x\right\}\right)=1,
$$

then for some $\alpha<\lambda$, 


$$
\mu\left(\left\{x \in p_{k} \lambda: f(x)=\alpha\right\}\right)=1 .
$$

$\kappa$ is $\lambda$-supercompact if there is a normal measure on $p_{k} \lambda$ and $k$ is supercompact if it is $\lambda$-supercompact for all $\lambda \geqslant \kappa$.

We assume that the reader is familiar with the elementary ultrapower techniques. If $\mu$ is an $\aleph_{1}$-additive measure on a set $X, " j: V \rightarrow M=V^{X} / \mu$ " will always mean that $j$ is the canonical elementary embedding of the universe into the transitive collapse $M$ of the ultrapower of the universe with respect to $\mu$. We sometimes denote $M$ by " $j(V)$ ". We also refer to $j$ as "the elementary embedding associated with $\mu$ ". All elementary embeddings will be assumed to be with respect to the $\epsilon$-relation.

Let $\mu$ be a normal measure on $p_{\kappa} \lambda$ and $j_{\lambda}: V \rightarrow M_{\lambda} \simeq V^{p_{\kappa} \lambda} / \mu$. By a theorem of Solovay, $M_{\lambda}$ is closed under $\lambda^{\kappa}$-sequences and $j_{\lambda}(\kappa)>\lambda$ [7]. If $f$ is a function with domain $p_{\kappa} \lambda,{ }^{r} f^{\prime \mu}$ will always be the element of $M_{\lambda}$ that corresponds to the equivalence class of $f$ with respect to $\mu$. We omit the " $\mu$ " when no confusion results. If $c$ is in $M_{\lambda},\left\langle c_{x} ; x \in p_{k} \lambda\right\rangle$ will be some function such that $r\left(c_{x} ; x \in p_{\kappa} \lambda\right\rangle$ ? =c. Suppose $\kappa \leqslant \nu \leqslant \lambda$ and $q: p_{\kappa} \lambda \rightarrow p_{\kappa} \nu$ is such that $q(x)=$ $x \cap \nu$ for every $x \in p_{\kappa} \lambda$. Then the measure $q_{*}(\mu)$ defined on $p_{\kappa} \nu$ so that for every subset $A$ of $p_{\kappa} \nu, q_{*}(\mu)(A)=1$ iff $\mu\left(\left\{x \in p_{k} \lambda: q(x) \in A\right\}\right)=1$, is a normal measure on $p_{\kappa} \nu$ and is said to be the projection of $\mu$ on $p_{\kappa} \nu$. It is not difficult to prove that for every subset $A$ of $p_{\kappa} \nu, q_{*}(\mu)(A)=1$ iff $j_{\lambda}[\nu] \in j_{\lambda}(A)$. Let $j_{\nu}: V \rightarrow M_{\nu} \simeq V^{p_{\kappa} \nu} / q_{*}(\mu)$. Then there is an elementary embedding $k: M_{\nu} \rightarrow M_{\lambda}$ such that $k \circ j_{\nu}=j_{\lambda}$. In fact, for every $f: p_{\kappa} \nu \rightarrow V$,

$$
k\left(\ulcorner f]^{q *(\mu)}\right)=r_{f} \circ q^{\mu} \text {. }
$$

1. On the Silver forcing method. The reader is expected to know in detail the papers of Scott and Solovay on Boolean-valued models of set theory [8] and of Solovay and Tennenbaum on iterated Cohen extensions [12].

This section bristles with notational conventions so that no part is intelligible without a perusal of its predecessors. Subsection 1 concerns two-stage extensions, and subsection 2 concerns limit stages. Subsection 3 is devoted to properties of a very fine system. In subsection 4 we show how to preserve the axioms of ZFC in forcing with suitable classes of conditions.

Subsection 1. Two-stage extensions. If $R$ is any partially ordered set, $P_{R}$ will be the underlying set and $\leqslant_{R}$ will be the partial ordering on $P_{R}$, i.e., $R=$ $\left\langle P_{R} ; \leqslant_{R}\right\rangle$. We require that $\leqslant_{R}$ be so that for every $x$ and $y$ in $P_{R}$ if $x \leqslant_{R} y$ and $y \leqslant_{R} x$, then $x=y$. Two elements $p$ and $r$ of $P_{R}$ are incompatible iff there is no $s$ in $P_{R}$ so that $s \leqslant_{R} p$ and $s \leqslant_{R} r$. $R$ is separative if for every $p$ and $q$ in $P_{R}$, either $q \leqslant_{R} p$ or there is an $r$ in $P_{R}$ so that $r \leqslant_{R} q$ and $r$ is incompatible with $p$. If $R$ has a greatest element, it is unique and will be denoted by " $1_{R}$ ". 
Suppose $P$ is a partially ordered set and $\lambda$ is an ordinal. $P$ is $\lambda$-closed if for every descending $\lambda$-sequence $\left\langle p_{\alpha} ; \alpha<\lambda\right\rangle$ in $P$ (i.e., $p_{\beta} \leqslant{ }_{p} p_{\alpha}$ for all $\alpha \leqslant$ $\beta<\lambda$ ), there is a $p$ in $p_{p}$ such that $p \leqslant{ }_{p} p_{\alpha}$ for all $\alpha<\lambda$. $P$ is $\lambda$-directed closed if for every directed $\lambda$-sequence $\left\langle p_{\alpha} ; \alpha<\lambda\right\rangle$ in $P$ (i.e., for every $\alpha<\beta<\lambda$, there is a $\gamma<\lambda$ such that $p_{\gamma} \leqslant p p_{\alpha}$ and $p_{\gamma} \leqslant p p_{\beta}$ ) there is a $p$ in $P_{p}$ such that $p \leqslant \leqslant_{p}$ $p_{\alpha}$ for all $\alpha<\lambda$.

If $C$ is a Boolean algebra " $B_{C}$ " will denote the underlying set and " ${ }_{C}$ ", ". $c$ ", and "- $c$ " will denote the Boolean operations of join, meet, and complement respectively. $0_{C}$ and $1_{C}$ will be the zero and unit elements of $C$ and $\leqslant_{C}$ will be the relation on $B_{c}$ defined so that for $a_{0}$ and $a_{1}$ in $B_{c}, a_{0} \leqslant{ }_{c} a_{1}$ iff $a_{0}{ }_{c}{ }_{c}$ $\left(a_{1}\right)=0_{c}$. If $C$ is complete, $\Sigma_{c}$ and $\Pi_{c}$ will be the infinite join and the infinite meet, respectively.

Let $B$ and $C$ be complete Boolean algebras, and $i$ a complete embedding of $B$ into $C$. The projection $\pi$ of $C$ on $B$ with respect to $i$ is the map from $B_{C}$ into $B_{B}$ defined so that $\pi(c)=\Pi_{B}\left\{b \in B_{B}: c \leqslant_{C} i(b)\right\}$ for all $c$ in $B_{C}$.

Now suppose that $B$ is a complete Boolean algebra and that $P \subseteq B_{B}$ is a dense subset of $B$ (i.e., $0_{B} \notin P$ and for every $b \in B_{B}$, if $b \neq 0_{B}$, there is a $p \in P$ so that $\left.p \leqslant \leqslant_{B} b\right)$. Then $P=\left\langle P ; \leqslant_{B} \mid P\right\rangle$ is a separative partially ordered set.

Conversely, if $R$ is a separative partially ordered set, there is a canonical complete Boolean algebra $B(R)$ and a mapping []$: R \rightarrow B(R)$ so that the set $\left[P_{R}\right]=$ $\left\{[r]: r \in P_{R}\right\}$ is a dense subset of $B(R)$ and [ ] gives an isomorphism of $R$ with the partially ordered set $\left\langle\left[P_{R}\right] ; \leqslant_{B(R)} \mid\left[P_{R}\right]\right\rangle$.

We now describe [ ] and $B(R)$. For $p \in P_{R}$, let $[p]=\left\{q \in P_{R}: q \leqslant{ }_{R} p\right\}$. We work with the topology $\tau$ on $P_{R}$ generated by the family $\left\{[p]: p \in P_{R}\right\}$. $B_{B(R)}$ is the set of all regular open subsets of $P_{R}$. [See Halmos [2] for the relevant topological and Boolean algebra concepts.] With respect to $\tau$, a subset $b$ of $P$ is regular open iff $(\forall p \in b)\left(\forall q \in P_{R}\right)\left(q<_{R} p \rightarrow q \in b\right)$ and $\left(\forall p \in P_{R}\right)$ $\left(\left(\forall q \leqslant_{R} p\right)\left(\exists r \leqslant_{R} q\right)(r \in b) \rightarrow p \in b\right)$. For $b_{0}, b_{1} \in B_{B(R)}, b_{0}{ }_{B(R)} b_{1}=b_{0}$ $\cap b_{1}, b_{0}+_{B(R)} b_{1}=\left\{p \in P_{R}:(\forall q \leqslant R p)\left(\exists r \leqslant \leqslant_{R} q\right)\left(r \in b_{0} \cup b_{1}\right)\right\},-{ }_{B(R)} b_{0}=$ $\left\{p \in P_{R}:\left(\forall q \leqslant \leqslant_{R} p\right)\left(q \notin b_{0}\right)\right\}$, and $b_{0} \leqslant_{B(R)} b_{1}$ iff $b_{0} \subseteq b_{1}$.

Convention $X$. Henceforth we reserve the term "poset" for any separative partially ordered set with a greatest element. If $P_{i}$ is a poset, where $i$ is any subscript, we let $P_{i}, \leqslant_{i}, B_{i}, B_{i},{ }_{i},+_{i},-{ }_{i}, 0_{i}$, and $1_{i}$, be $P_{P_{i}}, \gtrless_{P_{i}}, B\left(P_{i}\right), B_{B\left(P_{i}\right)}$, ${ }_{B\left(P_{i}\right)},+_{B\left(P_{i}\right)},-_{B\left(P_{i}\right)}, 0_{B\left(P_{i}\right)}$, and $1_{B\left(P_{i}\right)}$ respectively. We also use " $\leqslant_{i}$ " to denote the relation $\leqslant_{B\left(P_{i}\right)}$ and omit the brackets from " $[p]$ " for all $p \in P_{P_{i}}$.

Now suppose that $C$ is a complete Boolean algebra and that $P$ is a dense subset of $C$ so that $1_{C} \in P$. We have noted that $P=\left\langle P ; \leqslant_{C} \mid P\right\rangle$ is a poset. There is a unique isomorphism $e: B(P) \rightarrow C$ such that $e(p)=p$ for all $p \in P$.

If $B$ is a complete Boolean algebra, $V^{(B)}$ will be the separated Boolean valued universe and the maps ${ }^{r}: V \rightarrow V^{(B)}$ and ${ }^{\wedge}: V^{(B)} \rightarrow V$ will be as in [12]. 
If $\phi\left(v_{0}, \ldots, v_{n}\right)$ is a formula of ZF and $x_{0}, \ldots, x_{n}$ are elements of $V^{(B)}$, $\left\|\phi\left(x_{0}, \ldots, x_{n}\right)\right\|^{(B)}$ is the Boolean value of the statement $\phi\left(x_{0}, \ldots, x_{n}\right)$. The superscript " $(B)$ " will be omitted whenever possible. Also whenever we say that in $V^{(B)}, \phi\left(x_{0}, \ldots, x_{n}\right)$ or that $V^{(B)} \vDash \phi\left(x_{0}, \ldots, x_{n}\right)$, we mean that

$$
\left\|\phi\left(x_{0}, \ldots, x_{n}\right)\right\|^{(B)}=1_{B} .
$$

If $u$ and $v$ are sets such that $v \subseteq V^{(B)}$ and $f$ is a function from $u$ into $v, f^{*}$ will be the unique element of $V^{(B)}$ so that

$$
1_{B}=\| f^{*} \text { is a function with domain } \check{u} \|^{(B)}
$$

and for every $x \in u,\left\|f^{*}(\check{x})=f(x)\right\|^{(B)}=1_{B}$.

Suppose that $B$ is a complete Boolean algebra and that in $V^{(B)}, D$ is a complete Boolean algebra. It is shown in [12] that $\widetilde{D}=\left\langle\hat{B}_{D} ; \hat{+}_{D}, \hat{\circ}_{D}, \hat{-}_{D}\right\rangle$ is a com. plete Boolean algebra and that there is a canonical complete embedding $i_{B D}$ of $B$ into $\widetilde{D}$ so that for all $b \in B_{B},\left\|i_{B D}(b)=1_{D}\right\|=b$ and $\left\|i_{B D}(b)=0_{D}\right\|=-{ }_{B} b$.

Let $i$ be $i_{B D}$ and let $\pi$ be the projection of $\widetilde{D}$ on $B$ with respect to $i$. We recall two important facts of [12].

0. Proposition. For every $b \in B_{B}$ and for every $x \in B_{\tilde{D}}, \pi(i(b) \cdot x)=$ i(b) $\cdot \pi(x)$.

1. Proposition. For every $b \in B_{B}$ and $x, y \in B_{\tilde{D}}, i(b) \cdot{ }_{\tilde{D}} x \leqslant_{\tilde{D}} i(b) \cdot{ }_{\tilde{D}} y$ iff $b \leqslant{ }_{B}\left\|x \leqslant{ }_{D} y\right\|$.

2. Proposition. For every $x \in B_{\widetilde{D}}, \pi(x)=\left\|x \neq 0_{0}\right\|$.

Proof. By Proposition 1, $i\left(\left\|x=0_{0}\right\|\right) \cdot \widetilde{D} x=0_{0}$. Then $x \leqslant \tilde{D} i\left(\left\|x \neq 0_{0}\right\|\right)$, since $\left\|x=0_{0}\right\|=-_{B}\left\|x \neq 0_{0}\right\|$.

Suppose $x \leqslant_{\tilde{D}} i(b)$. Then $\left\|x \neq 0_{0}\right\| \leqslant_{B}\left\|i(b) \neq 0_{0}\right\|$, and since $\left\|i(b) \neq 0_{0}\right\|$ $=b, i\left(\left\|x \neq 0_{0}\right\|\right) \leqslant_{B} b$.

3. Proposition. Suppose that in $V^{(B)}, P$ is a dense subset of $D$. Then the set $\left\{i(b) \cdot \tilde{D} p: b \in B_{B}, b \neq 0_{B}\right.$, and $\left.p \in \hat{P}\right\}$ is a dense subset of $\widetilde{D}$.

Proof. First note that if $p \in \hat{P}$ and $b \in B_{B}$ so that $b \neq 0_{B}$, then $i(b) \cdot \tilde{D}$ $p \neq 0_{\tilde{D}}$. This follows from the fact that $\left\|p \neq 0_{D}\right\|=1_{B}$ and from the fact that by Proposition $2, \pi(i(b) \cdot \tilde{D} p)=b \cdot_{B}\left\|p \neq 0_{D}\right\|=b$.

Suppose $x \in B_{\tilde{D}}$ so that $x \neq 0_{\tilde{D}}$. Then $0_{B}<_{B} b=\left\|x \neq 0_{0}\right\|$. Since $V^{(B)}$ $\vDash\left(x \neq 0_{D} \rightarrow(\exists p \in P)\left(p \leqslant_{D} x\right)\right)$, there is a $p \stackrel{P}{\in} \hat{P}_{\text {with }}^{B}\left\|x \neq 0_{D}\right\| \leqslant_{B}\left\|p \leqslant_{D} x\right\|$. By Proposition $1, i(b) \cdot \tilde{\delta} p \leqslant_{\tilde{D}} i(b) \cdot \tilde{\delta} x$, and by Proposition $2, i(b) \cdot \tilde{D} x=x$.

Now let $P_{0}$ be a poset and let $P_{1}$ be a poset in $V^{\left(B\left(P_{0}\right)\right)}$. Let $i$ be $i_{B\left(P_{0}\right) B\left(P_{1}\right)}$, and let $\pi$ be the projection of $B\left(P_{1}\right)$ on $B\left(P_{0}\right)$ associated with $i$. Define $P_{0} \widetilde{\otimes} P_{1}=\left\{u \in \hat{B}_{1}:\left(\exists p \in P_{0}\right)\left(\exists q \in \hat{P}_{1}\right)\left(u=i(p) \hat{\bullet}_{1} q\right)\right\}$. We abbreviate 
" $i(p) \hat{}_{1} q$ " to " $i(p) q$ " when no confusion seems likely. Proposition 4 will show that the representation of $u \in P_{0} \widetilde{\otimes} P_{1}$ as $i(p) \hat{i}_{1} q$ is not in general unique; that is, it is possible that for a $q^{\prime} \in \hat{P}_{1}$ distinct from $q, u=i(p) \hat{\bullet}_{1} q=i(p) \hat{\bullet}_{1} q^{\prime}$. However the $p$ is unique. For $i\left(p_{0}\right) q_{0}$ and $i\left(p_{1}\right) q_{1}$ in $P_{0} \widetilde{\otimes} P_{1}$, set $i\left(p_{0}\right) q_{0}$ $\leqslant p_{0} \otimes P_{1} i\left(p_{1}\right) q_{1}$ iff $i\left(p_{0}\right) q_{0} \hat{\leqslant}_{1} i\left(p_{1}\right) q_{1}$. Then the set $P_{0} \widetilde{\otimes} P_{1}=\left\langle P_{0} \widetilde{\otimes} P_{1}\right.$; $\left.\leqslant_{p_{0} \otimes p_{1}}\right\rangle$ is a poset in our sense of the, word with greatest element $i\left(1_{p_{0}}\right) 1_{P_{1}}$.

By Proposition 3, $P_{0} \widetilde{\otimes} P_{1}$ is a dense subset of $\widetilde{B}_{1}$. There is a unique isomorphism $e: B\left(P_{0} \widetilde{\otimes} P_{1}\right) \rightarrow \widetilde{B}_{1}$ so that for every $i(p) q \in P_{0} \widetilde{\otimes} P_{1}, e(i(p) q)=$ $i(p) q$.

4. Proposition. For every $i\left(p_{0}\right) q_{0}$ and $i\left(p_{1}\right) q_{1}$ in $P_{0} \widetilde{\otimes} P_{1}, i\left(p_{0}\right) q_{0}$ $\leqslant_{p_{0} \circledast p_{1}} i\left(p_{1}\right) q_{1}$ iff $p_{0} \leqslant p_{1}$ and $p_{0} \leqslant 0\left\|q_{0} \leqslant 1 q_{1}\right\|$.

Proof. Suppose $c_{0}, c_{1} \in \hat{B}_{1}$ are such that $\left\|c_{0} \neq 0_{1}\right\|=\left\|c_{1} \neq 0_{1}\right\|=1_{0}$. Let $b_{0}, b_{1} \in B_{0}$.

Suppose that $i\left(b_{0}\right) \hat{\bullet}_{1} c_{0} \hat{\kappa}_{1} i\left(b_{1}\right) \hat{\bullet}_{1} c_{1}$. Then $i\left(b_{0} \cdot_{0}-{ }_{0} b_{1}\right) \hat{i}_{1} c_{0}=0_{1}$. By Proposition 2, $\pi\left(c_{0}\right)=1_{0}$. So $b_{0} \cdot_{0}-{ }_{0} b_{1}=0_{0}$ and $b_{0} \leqslant_{0} b_{1}$. Then $i\left(b_{0}\right)$ $\hat{i}_{1} c_{0} \hat{\leqslant}_{1} i\left(b_{0}\right) \hat{i}_{1} c_{1}$, and by Proposition $1, b_{0} \leqslant\left\|c_{0} \leqslant_{1} c_{1}\right\|$. Conversely one may check that if $b_{0} \leqslant_{0} b_{1}$ and $b_{0} \leqslant\left\|c_{0} \leqslant 1 c_{1}\right\|$, then $i\left(b_{0}\right) \hat{\leftrightarrow}_{1} c_{0} \hat{\leqslant}_{1} i\left(b_{1}\right)$ $\hat{\cdot}_{1} c_{1}$. Note that $\pi\left(i\left(b_{0}\right) \hat{i}_{1} c_{0}\right)=b_{0} \cdot{ }_{0} \pi\left(c_{0}\right)=b_{0}$.

The proposition now follows from the fact that $\left(\forall q \in \hat{P}_{1}\right)\left(\left\|q \neq 0_{1}\right\|=\right.$ $\left.1_{0}\right)$.

We now digress (up to Proposition 5) to consider the special case when we are working with Gödel-Bernays class-set theory and the "poset" $P_{1}$ is a proper Boolean valued class of $V^{\left(B_{0}\right)}$. The operation $\widetilde{\otimes}$ as defined above will no longer work because we can not form $B\left(P_{1}\right)$ in $V^{\left(B_{0}\right)}$. However we will consider a similar operation, denote it by " $\widetilde{\otimes}$ " also, and show that Proposition 4 holds for this new construction.

Let $U$ be the $\check{V}$-generic subset of $\check{P}_{0}$ defined so that for every $x \in P_{0}$, $\|\check{x} \in U\|=x$ and $\|\check{x} \notin U\|=(-x)^{2}$. Select an element $a$ of $V^{\left(B_{0}\right)}$ so that $\left\|a \in P_{1}\right\|=0_{0}$. In $V^{\left(B_{0}\right)}$ define $P_{1}^{*}=\left\langle P_{1}^{*}, \leqslant^{*}\right\rangle$ so that $P_{1}^{*}=P_{1} \cup\{a\}$ and for every $x, y \in P_{1}^{*}, x \leqslant_{1}^{*} y$ iff $x=a$ or $x \neq a, y \neq a$, and $x \leqslant_{1} y$. In $V^{\left(B_{0}\right)}, P_{1}^{*}$ is a partially ordered class with least element $a$. In $V^{\left(B_{0}\right)}$ define $f: \check{P}_{0} \times P_{1} \rightarrow P_{1}^{*}$ so that $\left(\forall x \in \check{P}_{0}\right)\left(\forall y \in P_{1}\right)(f(x, y)=y$ if $x \in U$ and $f(x, y)=a$ if $x \notin U)$.

Let $P_{0} \widetilde{\otimes} P_{1}=\left\{x \in V^{\left(B_{0}\right)}: \exists p \in P_{0} \exists q \in \hat{P}_{1}\right.$ so that $\left.1_{0}=\|f(\check{p}, q)=x\|\right\}$. For $p_{0}, p_{1} \in P_{0}$ and $q_{0}, q_{1} \in \hat{P}_{1}$, set $f\left(\check{p}_{0}, q_{0}\right) \leqslant p_{0} \otimes p_{1} f\left(\check{p}_{1}, q_{1}\right)$ iff $\| f\left(\check{p}_{0}, q_{0}\right)$ $\leqslant_{1}^{*} f\left(\check{p}_{1}, q_{1}\right) \|=1_{0}$. This holds iff $p_{0} \leqslant p_{1}$ and $p_{0} \leqslant\left\|q_{0} \leqslant_{1} q_{1}\right\|$. Let $P_{0}$ $\widetilde{\otimes} P_{1}=\left\langle P_{0} \widetilde{\otimes} P_{1} ; \leqslant p_{0} \widetilde{\otimes} P_{1}\right\rangle$. Clearly if $P_{1}$ is a set in $V^{\left(B_{0}\right)}$, then $P_{0} \widetilde{\otimes} P_{1}$ with respect to the former $\widetilde{\otimes}$-operation is isomorphic to $P_{0} \widetilde{\otimes} P_{1}$ with respect to 
this latter $\widetilde{\otimes}$-operation. For this revised $\widetilde{\otimes}$-operation the map that sends $p \in P_{0}$ into $f\left(\check{p}, 1_{1}\right)$ is an embedding of $P_{0}$ into $P_{0} \widetilde{\otimes} P_{1}$. Proposition 6 will hold for both versions of $\widetilde{\otimes}$ and for the case when $P_{1}$ is a class of $V^{\left(B_{0}\right)}$.

5. Proposition. (i) Suppose $P_{0}$ is $\lambda$-closed and in $V^{\left(B\left(P_{0}\right)\right)}, P_{1}$ is $\grave{\lambda}$-closed. Then $P_{0} \widetilde{\otimes} P_{1}$ is $\lambda$-closed.

(ii) Suppose $P_{0}$ is $\lambda$-directed closed and in $V^{\left(B\left(P_{0}\right)\right)}, P_{1}$ is $\check{\lambda}$-directed closed. Then $P_{0} \widetilde{\otimes} P_{1}$ is $\lambda$-directed closed.

Proof. (i) Let $\left\langle i\left(p_{\alpha}\right) q_{\alpha} ; \alpha<\lambda\right\rangle$ be a descending sequence in $P_{0} \widetilde{\otimes} P_{1}$. By Proposition $4,\left\langle p_{\alpha} ; \alpha<\lambda\right\rangle$ is a $\lambda$-descending sequence in $P_{0}$. There is a $p \in P_{0}$ with $p \leqslant_{0} p_{\alpha}$ for all $\alpha<\lambda$. Since for $\alpha<\beta<\lambda, p_{\beta} \leqslant_{0}\left\|q_{\beta} \leqslant_{1} q_{\alpha}\right\|$,

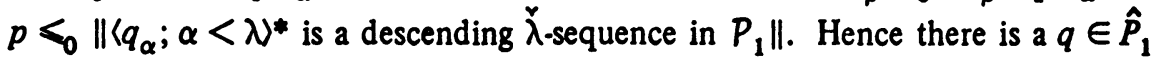
such that $p \leqslant_{0}\left\|q<_{1} q_{\alpha}\right\|$ for all $\alpha<\lambda$. Then $i(p) q \leqslant_{p_{0} \circledast p_{1}} i\left(p_{\alpha}\right) q_{\alpha}$ for all $\alpha<\lambda$.

(ii) Let $\left\langle i\left(p_{\alpha}\right) q_{\alpha} ; \alpha<\lambda\right\rangle$ be a $\lambda$-directed sequence in $P_{0} \widetilde{\otimes} P_{1}$. Then $\left\langle p_{\alpha}\right.$; $\alpha<\lambda$ is a $\lambda$-directed sequence in $P_{0}$, and there is a $p \in P_{0}$ so that $P<_{0} p_{\alpha}$ for every $\alpha<\lambda$.

For $\alpha<\beta<\lambda$, there is a $\gamma<\lambda$ so that $p \leqslant_{0} \| q_{\gamma} \leqslant_{1} q_{\alpha}$ and $q_{\gamma} \leqslant_{1} q_{\beta} \|$.

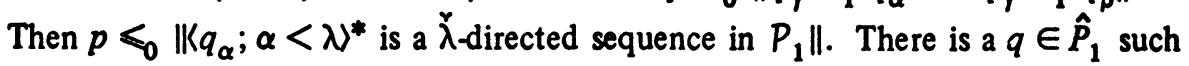
that $p \leqslant\left\|q \leqslant q_{\alpha}\right\|$ for all $\alpha<\lambda$. Hence $i(p) q \leqslant p_{0} \otimes p_{1} i\left(p_{\alpha}\right) q_{\alpha}$ for all $\alpha<\lambda$.

The following observation concerning the proof of part (i) will be needed in Subcase II' of the proof of Proposition 10. Namely, for any $p$ in $P_{0}$ so that $p \leqslant_{0} p_{\alpha}$ for all $\alpha<\lambda$ there is a $q$ in $\hat{P}_{1}$ so that $i(p) q \leqslant_{p_{0} \otimes p_{1}} i\left(p_{\alpha}\right) q_{\alpha}$ for all $\alpha<\lambda$. In other words, if $\left\langle r_{\alpha} ; \alpha<\lambda\right\rangle$ is a descending sequence in $P_{0} \widetilde{\otimes} P_{1}$ and $p \in P_{0}$ is so that $p<\pi\left(r_{\alpha}\right)$ for all $\alpha<\lambda$, then there is an $r \in P_{0} \otimes P_{1}$ so that $\pi(r)=p$ and $r \leqslant p_{0} \circledast p_{1} r_{\alpha}$ for all $\alpha<\lambda$.

In closing this subsection we mention an analogue of the "product lemma". The proof and the definitions of some of the concepts involved are to be found in [3].

Let $M$ be a countable standard model of ZFC and in $M$, let $P$ be a poset and $B(P)$ the canonical complete Boolean algebra associated with $P$. Suppose that $G$ is an $M$-generic subset of $P$. Then $U(G)=\left\{b \in B_{B(P)}:(\exists p \in G)(p \in b)\right\}$ is an M-generic ultrafilter on $B(P)$. Conversely, if $U$ is an M-generic ultrafilter on $B(P), G(U)=\left\{p \in P_{p}: p \in U\right\}$ is an M-generic subset of $P$.

If $U$ is an $M$-generic ultrafilter on $B(P), i_{U}$ will be the interpretation of $M^{B(P)}$ with respect to $U$. $i_{U}$ has the property that for all $x \in M^{B(P)}, i_{U}(x)=$ $\left\{i_{U}(y): y \in M^{B(P)}\right.$ and $\left.\|y \in x\| \in U\right\}$. 
Suppose that the discussion concerning $P_{0}$ and $P_{1}$ took place in $M$.

6. Proposition. (i) Let $G_{0}$ be an M-generic subset of $P_{0}$ and $G_{1}$ an $M\left[G_{0}\right]$-generic subset of $i_{U\left(G_{0}\right)}\left(P_{1}\right)$. Then $G=\left\{i(p) q: p \in G_{0}\right.$ and $i_{U\left(G_{0}\right)}(q) \in$ $G_{1}$ \} is an M-generic subset of $P_{0} \approx P_{1}$.

(ii) Let $G$ be an M-generic subset of $P_{0} \otimes P_{1}$. Then $G_{0}=\left\{p \in P_{0} ; i(p) 1_{1}\right.$ $\in G\}$ is an M-generic subset of $P_{0} . G_{1}=\left\{i_{U\left(G_{0}\right)}(q): i\left(1_{0}\right) q \in G\right\}$ is an $M\left[G_{0}\right]$ generic subset of $i_{U\left(G_{0}\right)}\left(P_{1}\right)$.

Subsection 2. Limit stages.

7A. Definition. Let $P$ and $R$ be posets. A map $i$ from $B(P)$ into $B(R)$ is fine iff the following conditions obtain.

I. $i$ is a complete embedding of $B(P)$ into $B(R)$ so that $i\left[P_{p}\right] \subseteq P_{R}$.

II. If $\pi$ is the projection of $B(R)$ on $B(P)$ associated with $i$, then $\pi\left[P_{R}\right]=$ $P_{P}$

III. If $p \in P_{R}$ and $q \in P_{P}$ are so that $q \leqslant_{p} \pi(p)$, then $i(q){ }_{B(R)} p \in P_{R}$.

Note that if $i$ is a fine map from $B(P)$ into $B(R)$, then since $P$ is a dense subset of $B(P)$ and $i$ is complete, the values of $i$ on $B(P)$ are uniquely determined by its values on $P$.

Remark (Solovay). The composition of fine maps is fine. For suppose that $P_{0}, P_{1}$, and $P_{2}$ are posets and that $i_{01}: B_{0} \rightarrow B_{1}$ and $i_{12}: B_{1} \rightarrow B_{2}$ are fine maps. Let $\pi_{21}: B_{2} \rightarrow B_{1}$ be the projection with respect to $i_{12}$ and $\pi_{10}: B_{1}$ $\rightarrow B_{0}$ the projection with respect to $i_{01}$. Set $i_{02}=i_{12} \circ i_{01}$, and let $\pi_{20}: B_{2} \rightarrow B_{0}$ be the projection with respect to $i_{02}$. We show that $i_{02}$ is a fine map. Condition I is obvious and condition II follows easily from the fact that $\pi_{20}=\pi_{10} \circ \pi_{21}$. To check for condition III, let $p \in P_{2}$ and $q \in P_{0}$ be so that $q \leqslant_{0} \pi_{20}(p)$. Since $i_{01}$ and $i_{12}$ are fine, $i_{01}(q) \cdot{ }_{1} \pi_{21}(p) \in P_{1}$ and $i_{12}\left(i_{01}(q) \cdot_{1} \pi_{21}(p)\right) \cdot_{2} p \in P_{2}$. But $i_{12}\left(i_{01}(q) \cdot{ }_{1} \pi_{21}(p)\right) \cdot{ }_{2} p=i_{02}(q) \cdot{ }_{2} i_{12}\left(\pi_{21}(p)\right) \cdot{ }_{2} p$, which is equal to $i_{02}(q)$ ${ }_{2} p$ since $p \leqslant_{2} i_{12}\left(\pi_{21}(p)\right)$ by the definition of $\pi_{21}$.

7B. Definition. Let $x$ be a nonempty class of ordinals. A sequence of posets $\left\langle P_{\beta} ; \beta \in x\right\rangle$ and a sequence of maps $\left\langle i_{\gamma \delta} ; \gamma, \delta \in x\right.$ and $\left.\gamma \leqslant \delta\right\rangle$ constitute a fine $x$-system iff for all $\gamma, \delta$, and $\eta$ in $x$ so that $\gamma \leqslant \delta \leqslant \eta$ :

(a) $i_{\gamma \delta}$ is a fine map from $B_{\gamma}$ into $B_{\delta}$, and $i_{\gamma \gamma}$ is the identity.

(b) $i_{\gamma \eta}=i_{\delta \eta} \circ i_{\gamma \delta}$.

We now consider three methods for extending the fine $x$-system $\left\langle P_{\beta} ; \beta \in x\right\rangle$ and $\left\langle i_{\gamma \delta} ; \gamma \leqslant \delta\right.$ and $\left.\gamma, \delta \in x\right\rangle$. For simplicity we assume that $x=\alpha$ for some ordinal $\alpha>0$. For $\gamma \leqslant \delta<\alpha$, let $\pi_{\delta \gamma}$ be the projection of $B_{\delta}$ on $B_{\gamma}$ with respect to $i_{\gamma \delta}$. 
Suppose that $\alpha=\beta+1$ and that in $V^{\left(B_{\beta}\right)}, R$ is a poset. Let $P_{\alpha}=P_{\beta} \widetilde{\otimes} R$, $e: B\left(P_{\alpha}\right) \rightarrow B(R)$ the canonical isomorphism, and set $i_{\beta \alpha}=e^{-1} \circ i_{B_{\beta} B(R)}$. For $\gamma<\beta$, let $i_{\gamma \alpha}=i_{\beta \alpha} \circ i_{\gamma \beta}$ and for $\gamma=\alpha$, let $i_{\gamma \alpha}$ be the identity restricted to $B_{\alpha}$. It is easy to check that the two pairs $\left\langle P_{\beta} ; \beta<\alpha+1\right\rangle$ and $\left\langle i_{\gamma \delta} ; \gamma \leqslant \delta<\alpha+1\right\rangle$ constitute a fine $\alpha+1$-system.

Now suppose that $\alpha$ is a limit ordinal. We will show that by letting $P_{\alpha}$ be the direct limit of $\left\langle P_{\beta} ; \beta<\alpha\right\rangle$ with respect to the embeddings $\left\langle i_{\gamma \delta} \mid P_{\gamma} ; \gamma \leqslant \delta<\alpha\right\rangle$ or the inverse limit of $\left\langle P_{\beta} ; \beta<\alpha\right\rangle$ with respect to the poset homomorphisms $\left\langle\pi_{\delta \gamma} \uparrow P_{\delta} ; \gamma \leqslant \delta<\alpha\right\rangle$, we can extend the above $\alpha$-system to a fine $\alpha+1$-system.

8. Proposition. Let $P_{\alpha}$ be the direct limit of the posets $\left\langle P_{\beta} ; \beta<\alpha\right\rangle$ with respect to the embeddings $\left\langle i_{\gamma \delta} \mid P_{\gamma} ; \gamma \leqslant \delta<\alpha\right\rangle$. For every $\beta \leqslant \alpha$, the canonical monomorphism of $P_{\beta}$ into $P_{\alpha}$ extends to a (unique) fine map $i_{\beta \alpha}$ from $B\left(P_{\beta}\right)$ into $B\left(P_{\alpha}\right)$ so that the pair $\left\langle P_{\beta} ; \beta \leqslant \alpha\right\rangle$ and $\left\langle i_{\gamma \delta} ; \gamma \leqslant \delta \leqslant \alpha\right\rangle$ constitute a fine $\alpha+1$ system.

Proof. We first recall the direct limit construction. Without loss of generality assume that the $P_{\beta}$ 's for $\beta<\alpha$ are disjoint.

For $x, y \in \bigcup_{\beta<\alpha} P_{\beta}$, let $\sigma(x)$ be the unique $\eta<\alpha$ such that $x \in P_{\eta}$ and define $x \sim y$ iff $(\exists \delta \geqslant \max (\sigma(x), \sigma(y)))\left(i_{\sigma(x) \delta}(x)=i_{\sigma(y) \delta}(y)\right)$. $\sim$ is an equivalence relation. Let $P_{\alpha}$ be the set of equivalence classes and for $x \in \bigcup_{\beta<\alpha} P_{\beta}$,

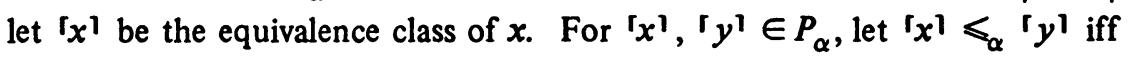
$(\exists \delta \geqslant \max (\sigma(x), \sigma(y)))\left(i_{\sigma(x) \delta}(x) \leqslant_{\delta} i_{\sigma(y) \delta}(y)\right)$. Set $P_{\alpha}=\left\langle P_{\alpha} ; \leqslant_{\alpha}\right\rangle$. The reader should verify that $P_{\alpha}$ is a poset in the sense of Convention $X$. For $\beta<\alpha$, the canonical monomorphism from $P_{\beta}$ into $P_{\alpha}$ is the function that maps every element of $P_{\beta}$ into its equivalence class.

A routine check establishes that for $\beta<\alpha$ the map $i_{\beta \alpha}: B\left(P_{\beta}\right) \rightarrow B\left(P_{\alpha}\right)$ defined for $a \in B_{\beta}$ by $i_{\beta \alpha}(a)=\left\{{ }^{\prime} p^{]}: p \in \bigcup_{\gamma<\alpha} P_{\gamma}\right.$ and $(\exists \delta \geqslant \sigma(p))\left(i_{\sigma(p) \delta}(p)\right.$ $\left.\left.\leqslant_{\delta} i_{\beta \delta}(a)\right)\right\}$ is a complete embedding extending the canonical monomorphism from $P_{\beta}$ into $P_{\alpha}$. [Note: one first shows that $i_{\beta \alpha}(a)$ is regular open and then that $i_{\beta \alpha}\left(-{ }_{\beta} a\right)=-{ }_{\alpha} i_{\beta \alpha}(a)$. It is obvious that $i_{\beta \alpha}\left(\bigcap_{\eta<v} a_{\eta}\right)=\bigcap_{\eta<v} i_{\beta \alpha}\left(a_{\eta}\right)$ for any sequence $\left\langle a_{\eta} ; \eta\langle v\rangle\right.$ of elements of $B_{\beta}$.]

Note that for $\beta, \gamma<\alpha$ and $a \in B_{\beta}$,

$$
\pi_{\alpha \gamma}\left(i_{\beta \alpha}(a)\right)= \begin{cases}a & \text { if } \gamma=\beta, \\ i_{\beta \gamma}(a) & \text { if } \gamma>\beta, \\ \pi_{\beta \gamma}(a) & \text { if } \gamma<\beta .\end{cases}
$$

Fix $\beta<\alpha$. To see that $i_{\beta \alpha}$ is fine note that property II (of a fine map) of $i_{\beta \alpha}$ follows from the preceding remark and from properties I and II of the $i_{\gamma \delta}$ 's 
for $\gamma<\delta<\alpha$. Property III follows from the same property of the $i_{\gamma \delta}$ 's for $\gamma \leqslant \delta<\alpha$.

We remark without proof that in the above construction, $B\left(P_{\alpha}\right)$ is isomorphic to the completion of the direct limit of the Boolean algebras $\left\langle B\left(P_{\beta}\right) ; \beta<\alpha\right\rangle$ with respect to the embeddings $\left\langle i_{\gamma \delta} ; \gamma \leqslant \delta<\alpha\right\rangle$.

Let us now consider the inverse limit construction. Define $P_{\alpha}=\{f \in$ $\Pi_{\beta<\alpha} P_{\beta}: \pi_{\delta \gamma}(f(\delta))=f(\gamma)$ for all $\left.\gamma \leqslant \delta<\alpha\right\}$. For $f$ and $g$ in $P_{\alpha}$, set $f \leqslant_{\alpha} g$ iff $f(\beta) \leqslant_{\beta} g(\beta)$ for every $\beta<\alpha$. The inverse limit of the posets $\left\langle P_{\beta} ; \beta<\alpha\right\rangle$ with respect to the poset homomorphisms $\left\langle\pi_{\delta \gamma} \uparrow P_{\delta} ; \gamma \leqslant \delta<\alpha\right\rangle$ is the partially ordered set $P_{\alpha}=\left\langle P_{\alpha} ; \xi_{\alpha}\right\rangle$.

The following property of $P_{\alpha}$ is needed in the proof of Proposition 9 and in the proof that $P_{\alpha}$ is separative.

Fix $\beta<\alpha$. Let $f \in P_{\alpha}$ and $q \in P_{\beta}$ be so that $q \leqslant_{\beta} f(\beta)$. Then there is a $g \in P_{\alpha}$ so that $g \leqslant_{\alpha} f$ and $g(\beta)=q . g$ is defined so that for $\gamma<\alpha$

$$
g(\gamma)= \begin{cases}\pi_{\beta \gamma}(q) & \text { if } \gamma<\beta . \\ i_{\beta \gamma}(q) \cdot{ }_{\gamma} f(\gamma) & \text { if } \gamma \geqslant \beta .\end{cases}
$$

To see that $g \in P_{\alpha}$ fix $\gamma<\delta<\alpha$. If $\delta \leqslant \beta$, then $\pi_{\delta \gamma}(g(\delta))=g(\gamma)$. If $\gamma \geqslant \beta$, then $\pi_{\delta \gamma}\left(i_{\beta \delta}(q) \cdot \delta f(\delta)\right)=i_{\beta \gamma}(q) \cdot{ }_{\gamma} \pi_{\delta \gamma}(f(\delta))=i_{\beta \gamma}(q) \cdot{ }_{\gamma} f(\gamma)$. If $\gamma<$ $\beta<\delta$, use the fact that $\pi_{\delta \gamma}=\pi_{\beta \gamma} \circ \pi_{\delta \beta}$. Clearly $g \leqslant_{\alpha} f$ and $g(\beta)=q$.

We now show that $P_{\alpha}$ is separative. Let $f_{0}, f_{1} \in P_{\alpha}$ be so that $f_{1} f_{\alpha} f_{0}$. Then there is a $\beta<\alpha$ so that $f_{1}(\beta) \$_{\beta} f_{0}(\beta)$. Since $P_{\beta}$ is separative there is a $q \in P_{\beta}$ so that $q \leqslant_{\beta} f_{1}(\beta)$ and $q$ is incompatible with $f_{0}(\beta)$. Let $g \in P_{\alpha}$ be so that $g \leqslant_{\alpha} f_{1}$ and $g(\beta)=q$. Then $g$ is incompatible with $f_{0}$.

9. Proposition. For every $\beta \leqslant \alpha$, there is a canonical fine map $i_{\beta \alpha}$ from $B\left(P_{\beta}\right)$ into $B\left(P_{\alpha}\right)$ so that the sequences $\left\langle P_{\beta} ; \beta \leqslant \alpha\right\rangle$ and $\left\langle i_{\gamma \delta} ; \gamma \leqslant \delta \leqslant \alpha\right\rangle$ form a fine $\alpha+1$ system.

Proof. Fix $\beta<\alpha$. Define $i_{\beta \alpha}: B\left(P_{\beta}\right) \rightarrow B\left(P_{\alpha}\right)$ so that for $a \in B_{\beta}, i_{\beta \alpha}(a)=$ $\left\{f \in P_{\alpha}: f(\beta) \in a\right\}$. It is routine to check that $i_{\beta \alpha}\left[P_{\beta}\right] \subseteq P_{\alpha}$. We show that it is a complete embedding.

To see that $i_{\beta \alpha}(a)$ is regular open first note that it is open. Then let $f \in P_{\alpha}$ be so that $\left(\forall g \leqslant_{\alpha} f\right)\left(\exists h \leqslant_{\alpha} g\right)(h(\beta) \in a)$. Suppose that $q \in P_{\beta}$ is so that $q \leqslant_{\beta}$ $f(\beta)$. By the property we established for $P_{\alpha}$, there is a $g \in P_{\alpha}$ so that $g \leqslant_{\alpha} f$ and $g(\beta)=q$. So there is an $h \in P_{\alpha}$ with $h \leqslant_{\alpha} g$ and $h(\beta) \in a$. Since $a$ is regular open, it follows that $f(\beta) \in a$ and hence that $f \in i_{\beta \alpha}(a)$.

One shows that $i_{\beta \alpha}\left(-_{\beta} a\right)=-{ }_{\alpha} i_{\beta \alpha}(a)$ by the same method. It is obvious that $i_{\beta \alpha}\left(\bigcap_{\eta<v} a_{\eta}\right)=\bigcap_{\eta<v} i_{\beta \alpha}\left(a_{\eta}\right)$. This suffices to prove that $i_{\beta \alpha}$ is a complete embedding. 
Note that for $f \in P_{\alpha}, \pi_{\alpha \beta}(f)=f(\beta)$. So $i_{\beta \alpha}$ has property II of a fine map. For property III, let $f \in P_{\alpha}$ and $q \leqslant \beta \leqslant(\beta)$. Define $g \in P_{\alpha}$ so that $g(\beta)=q$ and $g(\gamma)=i_{\beta \gamma}(q) \cdot{ }_{\gamma} f(\gamma)$ for all $\beta \leqslant \gamma<\alpha$. Then $i_{\beta \alpha}(q) \cdot{ }_{\alpha} f=g$.

In an unpublished paper concerning Suslin's hypothesis Jensen considers a construction for forcing conditions at a limit stage similar to the inverse limit construction defined above. Stephen Simpson inquired whether the two constructions are essentially equivalent.

Let us consider a precise formulation of the question. We continue to assume that $\alpha$ is a limit ordinal and that $P_{\alpha}$ is the inverse limit of $\left\langle P_{\beta} ; \beta<\alpha\right\rangle$ with respect to $\left\langle\pi_{\delta \gamma} \nmid P_{\delta} ; \gamma \leqslant \delta<\alpha\right\rangle$. For every $\beta<\alpha$ define a poset $R_{\beta}$ so that $P_{R_{\beta}}=B_{\beta}-\left\{0_{\beta}\right\}$ and $\leqslant_{R_{\beta}}=\leqslant_{\beta} \mid P_{R_{\beta}}$. Let $R_{\alpha}$ be the inverse limit of $\left\langle R_{\beta}\right.$; $\beta\langle\alpha\rangle$ with respect to the homomorphisms $\left\langle\pi_{\delta \gamma} \uparrow P_{R_{\delta}} ; \gamma \leqslant \delta<\alpha\right\rangle$. The question is whether $B\left(P_{\alpha}\right)$ is isomorphic to $B\left(R_{\alpha}\right)$. Solovay has shown that this is not always the case. However, if the $P_{\beta}$ 's (for $\beta<\alpha$ ) constitute a very fine system (to be defined below) and if in addition for every limit stage $\beta<\alpha, P_{\beta}$ is the inverse limit of its predecessors, then $B\left(P_{\alpha}\right)$ is isomorphic to $B\left(R_{\alpha}\right)$.

Subsection 3. Properties of very fine systems. We work with Gödel-Bernays class-set theory. The reader may translate arguments ostensibly requiring quantification over classes into proper class-set theory notation.

Definition. A fine system of posets $\left\langle P_{\alpha} ; \alpha\right.$ an ordinal $\rangle$ and embeddings $\left\langle i_{\gamma \delta} ; \gamma \leqslant \delta\right\rangle$ is very fine iff there is a sequence of sets $\left\langle P_{\gamma \gamma+1} ; \gamma\right.$ an ordinal so that for every ordinal $\gamma, P_{\gamma \gamma+1}$ is a poset in $V^{(B)}$, and the following conditions obtain.

I. If $\alpha=0$, then $P_{\alpha}=\langle\{0\} ;=\rangle$.

II. If $\alpha=\beta+1$, then $P_{\alpha}=P_{\beta} \widetilde{\otimes} P_{\beta \beta+1}$. Let $e$ be the canonical isomorphism from $B_{\alpha}$ into $\widetilde{B}_{\beta \alpha}$. Then $i_{\beta \alpha}=e^{-1} \circ i_{B_{\beta} \widetilde{B}_{\beta \alpha}}$ and for $\gamma \leqslant \beta, i_{\gamma \alpha}=i_{\beta \alpha}$ $\circ i_{\gamma \beta}$.

III. If $\alpha$ is an inaccessible cardinal so that for every $\gamma<\alpha,\left|P_{\gamma \gamma+1}\right|<\stackrel{\alpha}{\alpha}$ in $\left.V^{(B} \gamma\right)$, then $P_{\alpha}$ is either the direct limit or the inverse limit of $\left\langle P_{\beta} ; \beta<\alpha\right\rangle$, the former with respect to the embeddings $\left\langle i_{\gamma \delta} \nmid P_{\gamma} ; \gamma \leqslant \delta<\alpha\right\rangle$ and the latter with respect to the homomorphisms $\left\langle\pi_{\delta \gamma} \uparrow P_{\delta} ; \gamma \leqslant \delta<\alpha\right\rangle$. For every $\beta \leqslant \alpha, i_{\beta \alpha}$ is the canonical embedding of $B_{\beta}$ into $B_{\alpha}$.

IV. If $\alpha$ is a limit ordinal so that either $\alpha$ is not an inaccessible cardinal or for some $\gamma<\alpha,\left|P_{\gamma \gamma+1}\right| \geqslant \stackrel{\alpha}{\alpha}$ in $\left.V^{(B \gamma}\right)$, then $P_{\alpha}$ is the inverse limit of $\left\langle P_{\beta} ; \beta<\alpha\right\rangle$ with respect to the homomorphisms $\left\langle\pi_{\delta \gamma} \uparrow P_{\delta} ; \gamma \leqslant \delta<\alpha\right.$. For every $\beta<\alpha$, $i_{\beta \alpha}$ is the canonical embedding of $B_{\beta}$ into $B_{\alpha}$.

Condition $I$ is required only for notational convenience. Accordingly if 
$v<\alpha$ are ordinals and $x=\{\beta: v \leqslant \beta<\alpha\}$, we sometimes consider the now obvious concept of a very fine $x$-system.

Henceforth in this section we work with the very fine system $\left\langle P_{\alpha} ; \alpha\right.$ an ordinal $\rangle$ and $\left\langle i_{\gamma \delta} ; \gamma \leqslant \delta\right\rangle .\left\langle P_{\gamma \gamma+1} ; \gamma\right.$ an ordinal $\rangle$ is as in the definition of a very fine system, and for $\gamma \leqslant \delta, \pi_{\delta \gamma}$ is the projection of $B_{\delta}$ on $B_{\gamma}$ with respect to $i_{\gamma \delta}$.

10. Proposition. Let $\lambda$ be an ordinal so that for every cardinal $v$, if $P_{v}$ is the direct limit of its predecessors, then $\lambda<v$.

(i) Let $\alpha$ be an ordinal so that for every $\gamma\left\langle\alpha, V^{\left(B_{\gamma}\right)} \vDash\left(P_{\gamma \gamma+1}\right.\right.$ is $\check{\lambda}$ closed). Then $P_{\alpha}$ is $\lambda$-closed.

(ii) Let $\alpha$ be an ordinal so that for every $\lambda\left\langle\alpha, V^{\left(B_{\gamma}\right)} \vDash\left(P_{\gamma \gamma+1}\right.\right.$ is directed closed). Then $P_{\alpha}$ is $\lambda$-directed closed.

Proof. We only prove part (i) since the proof of part (ii) is essentially the same.

We show by induction on $\eta \leqslant \alpha$ that $P_{\eta}$ is $\lambda$-closed.

Case I. $P_{0}$ is obviously $\lambda$-closed.

Case II. For $\eta=\beta+1$, that $P_{\eta}$ is $\lambda$-closed follows from Proposition 4.5 and the induction hypothesis.

Case III. Suppose that $\eta$ is an inaccessible cardinal and that $P_{\eta}$ is the direct limit of its predecessors.

Let $\left\langle x_{\delta} ; \delta<\lambda\right\rangle$ be a decreasing sequence in $P_{\eta}$. By our assumption on $\lambda$, $\lambda<\eta$. Then there is a $\gamma\left\langle\eta\right.$ and a decreasing sequence $\left\langle p_{\delta} ; \delta<\lambda\right.$ in $P_{\gamma}$ so that $i_{\gamma \eta}\left(p_{\delta}\right)=x_{\delta}$ for all $\delta<\lambda$. By the induction hypothesis, there is a $p \in P_{\gamma}$ so that $p \leqslant_{\gamma} p_{\delta}$ for all $\delta<\lambda$. Then $i_{\gamma \eta}(p) \leqslant_{\eta} x_{\delta}$ for all $\delta<\lambda$.

Case IV. Suppose that $\eta$ is a limit ordinal and that $P_{\eta}$ is the inverse limit of its predecessors.

Let $\left\langle f_{\delta} ; \delta<\lambda\right\rangle$ be a decreasing sequence in $P_{\eta}$. By induction on $\beta \leqslant \eta$, define $a_{\beta} \in P_{\beta}$ so that $a_{\beta} \leqslant_{\beta} f_{\delta}(\beta)$ for all $\delta<\lambda$, and for all $\gamma_{0} \leqslant \gamma_{1} \leqslant \beta$, $\pi_{\gamma_{1} \gamma_{0}}\left(a_{\gamma_{1}}\right)=a_{\gamma_{0}}$ and if $i_{\gamma_{0} \gamma_{1}}\left(f_{\delta}\left(\gamma_{0}\right)\right)=f_{\delta}\left(\gamma_{1}\right)$ for all $\delta<\lambda$, then $i_{\gamma_{0} \gamma_{1}}\left(a_{\gamma_{0}}\right)=$ $a_{\gamma_{1}}$.

Subcase I'. For $\beta=0$, let $a_{\beta}=0$.

Subcase II'. Suppose $\beta=\gamma+1$. If $f_{\delta}(\gamma+1)=i_{\gamma \gamma+1}\left(f_{\delta}(\gamma)\right)$ for all $\delta<$ $\lambda$, let $a_{\beta}=i_{\gamma \gamma+1}\left(a_{\gamma}\right)$. Otherwise, by the proof of Proposition 5 and the induction hypotheses on $a_{\gamma}$, there is an $a_{\beta} \in P_{\beta}$ so that $a_{\beta} \leqslant_{\beta} f_{\delta}(\beta)$ for all $\delta<\lambda$ and $\pi_{\beta \gamma}\left(a_{\beta}\right)=a_{\gamma}$.

Subcase III'. If $\beta$ is an inaccessible cardinal and $P_{\beta}$ is the direct limit of its predecessors, then as in Case III of this proof, there is a $\gamma<\beta$ and a sequence 
$\left\langle p_{\delta} ; \delta<\lambda\right\rangle$ in $P_{\gamma}$ so that $i_{\gamma \beta}\left(p_{\delta}\right)=f_{\delta}(\beta)$ for all $\delta<\lambda$. Set $a_{\beta}=i_{\gamma \beta}\left(a_{\gamma}\right)$.

Subcase IV'. If $\beta$ is a limit ordinal and $P_{\beta}$ is the inverse limit of its predecessors, define $a_{\beta} \in P_{\beta}$ so that for all $\gamma<\beta, a_{\beta}(\gamma)=a_{\gamma}$.

11. Proposition. Let $v>0$ be an ordinal. For every $\alpha>v$ there is a set $P_{v \alpha}$ so that $V^{\left(B_{v}\right)} \vDash\left(P_{v \alpha}\right.$ is a poset $)$, and $P_{\alpha}$ is isomorphic to $P_{v} \widetilde{\otimes} P_{v \alpha}$.

Proof. We remind the reader of Convention X. By induction on $\alpha>v$ we will construct the set $P_{v \alpha}$, three mappings $e_{\alpha}, k_{\alpha}$, and $\pi_{\alpha}$, and for all $\beta$ so that $v<\beta \leqslant \alpha$, two sets $j_{\beta \alpha}$ and $\mu_{\alpha \beta}$ so that the following hold.

I. Let $x=\{\beta ; v<\beta \leqslant \alpha\}$. In $V^{\left(B_{v}\right)},\left\langle P_{v \beta} ; \beta \in x\right\rangle^{*}$ and $\left\langle j_{\gamma \delta} ; \gamma \leqslant \delta \text { in } x\right\rangle^{*}$ constitute a very fine $\check{x}$-system. For $\gamma \leqslant \delta$ in $x, V^{\left(B_{v}\right)} \vDash\left(\mu_{\delta \gamma}\right.$ is the projection of $B_{v \delta}$ on $B_{v \gamma}$ with respect to $\left.j_{\gamma \delta}\right)$. Also if $\gamma \in x$ is a limit ordinal, then $V^{\left(B_{v}\right)}$ $F\left(P_{v \gamma}\right.$ is the direct limit of its predecessors) or $V^{\left(B_{v}\right)} \vDash\left(P_{v \gamma}\right.$ is the inverse limit of its predecessors) according to whether $P_{\gamma}$ is the direct limit or the inverse limit of its predecessors respectively.

II. For $\beta \in x, e_{\beta}$ is an isomorphism from $B_{\beta}$ onto $\widetilde{B}_{v \beta}$ so that $e_{\beta}\left[P_{\beta}\right]=$ $P_{v} \widetilde{\otimes} P_{v \beta} . k_{\beta}$ is the embedding $i_{B_{v} B_{v \beta}}$ and $\pi_{\beta}$ is the associated projection. Let $\gamma \leqslant \delta$ be in $x$. The following diagrams commute.

Diagram A

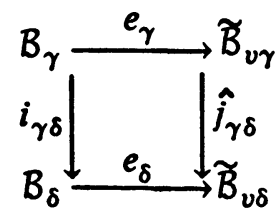

\section{Diagram B}

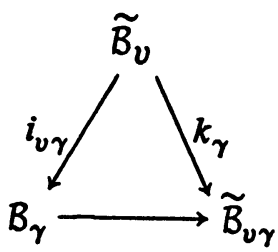

Note that by Lemma 2 of $\S 5.7$ of [12], $\hat{j}_{\gamma \delta} \circ k_{\gamma}=k_{\delta}$. Note also that the commutativity of Diagram B follows readily from the commutativity of Diagram B for $\gamma=v+1$ and from the commutativity of Diagram A.

Case I. $\alpha=v+1$. Then $P_{v \alpha}$ has been defined. Let $e_{\alpha}$ be the canonical isomorphism from $B_{\alpha}$ into $\widetilde{B}_{v \alpha}$ so that for $p \in P_{\alpha}, e_{\alpha}(p)=p$. Set $k_{\alpha}=i_{B_{v} B_{v \alpha}}$ and let $\pi_{\alpha}$ be the associated projection.

Case II. $\alpha=\beta+1$ and $\beta>v$. By induction hypotheses we have that $e_{\beta}$ : $B_{\beta} \rightarrow \widetilde{B}_{v \beta}=\left\langle\hat{B}_{v \beta} ; \hat{t}_{v \beta}, \hat{}_{v \beta}, \hat{}_{v \beta}\right\rangle$ is an isomorphism so that $e_{\beta}\left[P_{\beta}\right]=P_{v} \widetilde{\otimes} P_{v \beta}$. Also $P_{\alpha}=P_{\beta} \widetilde{\otimes} P_{\beta \alpha}$.

As in Lemma 5.3.1 of [12], there is a canonical isomorphism $\tau$ of $\widetilde{B}_{u p}$ - 
relational systems from $V^{\left(\widetilde{B}_{v \beta}\right)}$ onto $W^{\left(\widetilde{B}_{v \beta}\right)}$, where $W^{\left(\widetilde{B}_{v \beta}\right)}$ is essentially $V^{\left(B_{v \beta}\right)}$ computed in $V^{\left(B_{v}\right)}$, i.e., the underlying class of $W^{\left(\widetilde{B}_{v \beta}\right)}$ is the set $\left\{x \in V^{\left(B_{v}\right)}\right.$ : $V^{\left(B_{v}\right)} \vDash\left(x \in V^{\left(B_{v \beta}\right)}\right)$. Since $B_{\beta}$ and $\widetilde{B}_{v \beta}$ are isomorphic by $e_{\beta}$, there is a bijection $\sigma$ from $V^{\left(B_{\beta}\right)}$ onto $\left.W^{(\widetilde{B}} v \beta\right)$ so that if $b \in B_{\beta}, \varphi\left(v_{0}, \ldots, v_{n}\right)$ is a formula of ZF and $x_{0}, \ldots, x_{n}$ are elements of $V^{\left(B_{\beta}\right)}$, then

$$
\begin{aligned}
& b \leqslant_{\beta}\left\|\phi\left(x_{0}, \ldots, x_{n}\right)\right\|^{\left(B_{\beta}\right)} \text { iff } \\
& V^{\left(B_{v}\right)} \vDash\left(e_{\beta}(b) \leqslant_{v \beta}\left\|\phi\left(\sigma\left(x_{0}\right), \ldots, \sigma\left(x_{n}\right)\right)\right\|^{\left(B_{v \beta}\right)}\right) .
\end{aligned}
$$

Since $V^{\left(B_{\beta}\right)} \models\left(P_{\beta \alpha}=\left\langle p_{\beta \alpha} ; \leqslant_{\beta \alpha}\right\rangle\right.$ is a poset and $\left.B_{\beta \alpha}=B\left(P_{\beta \alpha}\right)\right), V^{\left(B_{v}\right)}$ $\vDash\left(1_{v \beta} \leqslant_{v \beta} \| \sigma\left(P_{\beta \alpha}\right)=\left\langle\sigma\left(P_{\beta \alpha}\right) ; \sigma\left(\leqslant_{\beta \alpha}\right)\right\rangle\right.$ is a poset and $\sigma\left(B_{\beta \alpha}\right)=$ $\left.B\left(\sigma\left(P_{\beta \alpha}\right)\right) \|^{\left(B_{v \beta}\right)}\right)$.

Working in $V^{\left(B_{v}\right)}$ : Let $P_{v \alpha}=P_{v \beta} \widetilde{\otimes} \sigma\left(P_{\beta \alpha}\right)$. Let $i=i_{P_{v \beta} \sigma\left(B_{\beta \alpha}\right)}$ and $\leqslant^{*}=\sigma\left(\leqslant_{\beta \alpha}\right)$. There is a canonical isomorphism $e: B_{v \alpha} \rightarrow \sigma\left(B_{\beta \alpha}\right)^{\sim}$ so that for $p \in P_{v \alpha}, e(p)=p$. Let $j_{\beta \alpha}: B_{v \beta} \rightarrow B_{v \alpha}$ be the map $e^{-1} \circ i$. For $\gamma \leqslant \alpha, j_{\gamma \alpha}$ and $\mu_{\alpha \gamma}$ are defined as usual.

Define $k_{\alpha}$ to be $i_{B_{v} B_{v \alpha}}$ and $\pi_{\alpha}$ the associated projection.

We are now ready to define $e_{\alpha}$. Let $u \in P_{\alpha}$. Then $u=i_{\beta \alpha}(p) q$ for some $p \in P_{\beta}$ and $q \in \hat{P}_{\beta \alpha}$. Let $e_{\beta}(p)=k_{\beta}(r) s$, where $r \in P_{v}$ and $s \in \hat{P}_{v \beta}$. Then $V^{(v)}$ $\mathrm{I}=\left(\sigma(q) \in \sigma\left(P_{\beta \alpha}\right)^{\wedge}\right.$ and $\left.s \in P_{v \beta}\right)$. Also $i(s) \sigma(q) \in P_{v \alpha}$. Define $e_{\alpha}(u)$ to be $k_{\alpha}(r)(i(s) \sigma(q))$.

We show that $e_{\alpha}$ is well defined and preserves order. For $n<2$, let $u_{n} \in$ $P_{\alpha}, u_{n}=i_{\beta \alpha}\left(p_{n}\right) q_{n}$, and $e_{\beta}\left(p_{n}\right)=k_{\beta}\left(r_{n}\right) s_{n}$ as above.

Suppose $u_{0} \leqslant{ }_{\alpha} u_{1}$. Then $p_{0} \leqslant{ }_{\beta} p_{1}$ and $p_{0} \leqslant \leqslant_{\beta}\left\|q_{0} \leqslant_{\beta \alpha} q_{1}\right\|^{\left(B_{\beta}\right)}$. Also $r_{0} \leqslant v r_{1}$ and $r_{0} \leqslant v\left\|s_{0} \leqslant v s_{1}\right\|^{\left(B_{v}\right)}$. By $(*)$ above in $V^{\left(B_{v}\right)}, k_{\beta}\left(r_{0}\right) s_{0} \leqslant v \beta$ $\left\|\sigma\left(q_{0}\right) \leqslant{ }^{*} \sigma\left(q_{1}\right)\right\|^{\left(B_{v \beta}\right)}$. Since $r_{0} \leqslant v\left\|k_{\beta}\left(r_{0}\right) s_{0}=s_{0}\right\|^{\left(B_{v}\right)}$, then

$$
r_{0} \leqslant v\left\|s_{0} \leqslant v\right\| \sigma\left(q_{0}\right) \leqslant * \sigma\left(q_{1}\right)\left\|^{\left(B_{v \beta}\right)}\right\|^{\left(B_{v}\right)} \text {. }
$$

Then $r_{0} \leqslant v\left\|i\left(s_{0}\right) \sigma\left(q_{0}\right) \leqslant_{v \alpha} i\left(s_{1}\right) \sigma\left(q_{1}\right)\right\|^{\left(B_{v}\right)}$ and $k_{\alpha}\left(r_{0}\right)\left(i\left(s_{0}\right) \sigma\left(q_{0}\right)\right) \hat{\leqslant}_{v \alpha}$ $k_{\alpha}\left(r_{1}\right)\left(i\left(s_{1}\right) \sigma\left(q_{1}\right)\right)$, i.e., $e_{\alpha}\left(u_{0}\right) \hat{\kappa}_{v \alpha} e_{\alpha}\left(u_{1}\right)$.

The chain of implications can be reversed: $e_{\alpha}\left(u_{0}\right) \hat{\leqslant}_{v \alpha} e_{\alpha}\left(u_{1}\right)$ implies that $u_{0} \leqslant u_{1}$.

It is not hard to check that $e_{\alpha}$ is surjective and that the inductive hypotheses hold.

Case III. $\alpha$ is a limit ordinal and $P_{\alpha}$ is the inverse limit of its predecessors.

In $V^{\left(B_{v}\right)}$, let $P_{v \alpha}$ be the inverse limit of the posets $\left(P_{v \beta} ; v<\beta<\alpha\right\rangle^{*}$ with respect to the poset homomorphisms $\left\langle\mu_{\delta \gamma} \uparrow P_{v \delta} ; v<\gamma \leqslant \delta\langle\alpha\rangle^{*}\right.$. For 
$v<\beta \leqslant \alpha$, let $j_{\beta \alpha}$ be in $V^{\left(B_{v}\right)}$ the canonical embedding of $B_{v \beta}$ into $B_{v \alpha}$, and let $\mu_{\alpha \beta}$ be in $V^{\left(B_{v}\right)}$, the canonical projection of $B_{v \alpha}$ on $B_{v \beta}$. Let $k_{\alpha}$ be $i_{B_{v} B_{v \alpha}}$ and $\pi_{\alpha}$ the associated projection.

Now let $u \in P_{\alpha}$ and $u(v)=\pi_{\alpha} u(v)=p$. Fix $\beta$ so that $v<\beta<\alpha$. Since by Diagram B, $\pi_{\beta}\left(e_{\beta}(u(\beta))\right)=p$, there is a $q_{\beta} \in \hat{P}_{v \beta}$ so that $e_{\beta}(u(\beta))=k_{\beta}(p) q_{\beta}$.

Set $q=\left\langle q_{\beta} ; v\langle\beta \leqslant \alpha\rangle^{*}\right.$ and $\mu=\left\langle\mu_{\delta \gamma} ; v<\gamma \leqslant \delta<\alpha\right\rangle^{*}$. Suppose $\gamma$ and $\delta$ are so that $v<\gamma \leqslant \delta<\alpha$. By Diagram $A$,

$$
1_{v}=\left\|\mu_{\delta \gamma}\left(k_{\delta}(p) q_{\delta}\right)=k_{\gamma}(p) q_{\gamma}\right\|^{(\mathrm{B} v)} \text {. }
$$

But $1_{v}=\left\|\mu_{\delta \gamma}\left(k_{\delta}(p) q_{\delta}\right)=k_{\gamma}(p) \mu_{\delta \gamma}\left(q_{\delta}\right)\right\|^{\left(B_{v}\right)}$, since

$$
1_{v}=\left\|j_{\gamma \delta}\left(k_{\gamma}(p)\right)=k_{\delta}(p)\right\|^{\left(B_{v}\right)} .
$$

So $p \leqslant_{v}\left\|\mu_{\delta \gamma}\left(q_{\delta}\right)=q_{\gamma}\right\|^{\left(B_{v}\right)}$. It follows that

$$
p \leqslant\left\|(\forall \gamma, \delta)(\check{v}<\gamma \leqslant \delta<\alpha) \rightarrow q(\gamma)=\mu(\gamma)=\mu_{\delta, \gamma}(q(\delta))\right\|^{\left(B_{v}\right)} .
$$

Define a function $U$ from $\check{B}_{v}$ into $B_{v}$ so that for $b \in B_{v}, U(\check{b})=b$. Then in $V^{\left(B_{v}\right)}, U$ is an ultrafilter on $\check{B}_{v}$; and for $b \in B_{v},\|\check{b} \in U\|=b$.

In $V^{\left(B_{v}\right)}$, define $f \in P_{v \alpha}$ so that for every $\beta$ with $\check{v}<\beta<\check{\alpha}, f(\beta)=1_{\beta}$ if $\check{p} \notin U$ and $f(\beta)=q(\beta)$ if $\check{p} \in U$. Set $e_{\alpha}(u)=k_{\alpha}(p) f$.

To show that $e_{\alpha}$ is well defined and preserves order, for $n<2$ let $u_{n} \in P_{\alpha}$ and let $p_{n}, q_{\beta}^{n}$ for $v<\beta<\alpha$, and $q^{n}$ and $f^{n}$ satisfy the obvious conditions.

Suppose $u_{0} \leqslant_{\alpha} u_{1}$ and fix $\beta$ so that $v<\beta<\alpha$. Then $p_{0} \leqslant v p_{1}$ and $p_{0} \leqslant v$ $\left\|q_{\beta}^{0} \leqslant_{v \beta} q_{\beta}^{1}\right\|$. Since for $n<2, p_{n}=\left\|\check{p}_{n} \in U\right\| \leqslant_{v} f_{n}(\check{\beta})=\check{q}_{\beta}^{n}$, it follows that $p_{0} \leqslant_{v}\left\|f_{0}(\check{\beta}) \leqslant_{\nu \beta} f_{1}(\check{\beta})\right\|$. Hence $p_{0} \leqslant_{v}\left\|f_{0} \leqslant_{\nu \alpha} f_{1}\right\|$ and $k_{\alpha}\left(p_{0}\right) f_{0} \hat{\leqslant}_{v \alpha}$ $k_{\alpha}\left(p_{1}\right) f_{1}$.

The converse implications also hold and show that $e_{\alpha}$ is injective and that $e_{\alpha}\left(u_{0}\right) \hat{\leqslant}_{v \alpha} e_{\alpha}\left(u_{1}\right)$ implies that $u_{0} \leqslant_{\alpha} u_{1}$.

To prove that $e_{\alpha}$ is surjective, let $k_{\alpha}(p) f \in P_{v} \widetilde{\otimes} P_{v \alpha}$. There is an $x \in$ $\Pi_{v<\beta<\alpha} \hat{P}_{v \beta}$ so that for all $\beta$ with $v<\beta<\alpha,\|f(\tilde{\beta})=x(\beta)\|=1_{v}$. Define $u \in$ $\Pi_{\beta<\alpha} P_{\beta}$ so that for $\beta<\alpha$

$$
u(\beta)= \begin{cases}\pi_{v \beta}(p) & \text { if } \beta \leqslant v, \\ e_{\beta}^{-1}\left(k_{\beta}(p) x(\beta)\right) & \text { if } \beta>v .\end{cases}
$$

An easy check using Diagrams A and B and the fact that for $v<\gamma \leqslant \delta<$ $\alpha, \hat{j}_{\gamma \delta} \circ k_{\gamma}=k_{\delta}$, establishes that $u \in P_{\alpha}$. Then $e_{\alpha}(u)=k_{\alpha}(p) f$.

Routine arguments show that the inductive hypotheses hold.

Case IV. $\alpha$ is an inaccessible cardinal and $P_{\alpha}$ is the direct limit of its predecessors. 
For the first time we make use of the fact that for every $\gamma<\alpha, V^{\left(B_{\gamma}\right)} \vDash$ $\left(\left|P_{\gamma \gamma+1}\right|<\stackrel{\alpha}{\alpha}\right)$. An easy induction on $\gamma<\alpha$ shows that $\left|B_{\gamma}\right|<\alpha$. In particular $\left|B_{v}\right|<\alpha$.

In $V^{\left(B_{v}\right)}$, let $P_{v \alpha}$ be the direct limit of the posets $\left(P_{v \beta} ; v<\beta<\alpha\right)^{*}$ with respect to the embeddings $\left\langle j_{\gamma \delta} \mid P_{\gamma} ; v<\gamma \leqslant \delta<\alpha\right\rangle^{*}$. For $\beta$ so that $v<\beta \leqslant \alpha$, let $j_{\beta \alpha}$ be in $V^{\left(B_{v}\right)}$ the canonical embedding of $B_{v \beta}$ into $B_{v \alpha}$, and let $\mu_{\alpha \beta}$ be in $V^{\left(B_{v}\right)}$ the canonical projection of $B_{v \alpha}$ on $B_{v \beta}$. Let $k_{\alpha}$ be $i_{B_{v} B_{v \alpha}}$ and $\pi_{\alpha}$ the associated projection.

Let $u \in P_{\alpha}$ and let $\eta$ be an ordinal greater than $v$ so that there is an $x \in P_{\eta}$ with $i_{\eta \alpha}(x)=u$. Set $e_{\alpha}(u)=\hat{j}_{\eta \alpha}\left(e_{\eta}(x)\right)$.

A check using Diagram A establishes that $e_{\alpha}$ is well defined and preserves order. That $e_{\alpha}$ is injective is also routine.

To show the surjectivity of $e_{\alpha}$, let $k_{\alpha}(p) z \in P_{v} \widetilde{\otimes} P_{v \alpha}$. Then

$$
1_{v}=\|(\exists \beta \in \check{\alpha})(\exists q \in P(\beta))(j(\beta)(q)=z)\|^{\left(B_{v}\right)} \text {, }
$$

where $j=\left\langle j_{\beta \alpha} ; v<\beta<\alpha\right\rangle^{*}$ and $P=\left\langle P_{\beta \alpha} ; v<\beta<\alpha\right\rangle^{*}$.

For $\beta$ so that $v<\beta<\alpha$, let

$$
g(\beta)=\|(\exists q \in P(\check{\beta}))(j(\stackrel{\gamma}{\beta})(q)=z)\|^{\left(B_{v}\right)} .
$$

Then for $v<\gamma \leqslant \delta<\alpha, g(\gamma) \leqslant{ }_{v} g(\delta)$. Since $\left|B_{v}\right|<\alpha$, there is a $\beta<\alpha$ so that $g(\beta)=1_{v}$. It follows that there is a $q \in \hat{P}_{v \beta}$ with $\hat{j}_{\beta \alpha}(q)=z$. Then

$$
e_{\alpha}\left(i_{\beta \alpha}\left(e_{\beta}^{-1}\left(k_{\beta}(p) q\right)\right)\right)=k_{\alpha}(p) z
$$

Routine arguments show that the inductive hypotheses hold.

12. Corollary. Let $\lambda$ and $\alpha>v>0$ be ordinals so that for every cardinal $\nu$ with $\alpha \geqslant \nu>v$, if $P_{\nu}$ is the direct limit of its predecessors, then $\lambda<\nu$. Suppose that for every $\beta \geqslant v$ with $\beta<\alpha, P_{\beta \beta+1}$ is $\lambda$-closed in $V^{\left(B_{\beta}\right)}$. Then the $P_{v \alpha}$ constructed in the proof of 11 has the additional property that in $V^{\left(B_{v}\right)}$,

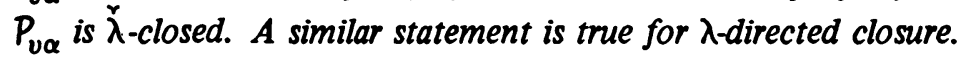

Proof. First note that if $v$ is a cardinal so that $\alpha \geqslant \nu>v$ and so that in $V^{\left(B_{v}\right)}, P_{v \nu}$ is the direct limit of its predecessors, then $\lambda<\nu$.

Now fix $\beta \geqslant v$ so that $\beta<\alpha$. If $\beta=v$, then by assumption, $V^{\left(B_{v}\right)} \vDash$ $\left(P_{v v+1}\right.$ is $\lambda$-closed). Suppose that $\beta>v$. Consider Case II in the proof of Proposition 10. We have $\sigma: V^{\left(B_{v}\right)} \leftrightarrow W^{\left(\widetilde{B}_{v \beta}\right)}$, and in $V^{\left(B_{v}\right)}, P_{v \beta+1}=P_{v \beta} \widetilde{\otimes} \sigma\left(P_{\beta \beta+1}\right)$. Since by assumption $V^{\left(B_{\beta}\right)} \vDash\left(P_{\beta \beta+1}\right.$ is $\left.\check{\lambda}_{\text {-closed }}\right)$, then in $V^{\left(B_{v}\right)}, V^{\left(B_{v \beta}\right)} \vDash$ $\left(\sigma\left(P_{\beta \beta+1}\right)\right.$ is $\check{\lambda}$-closed). [Note. $\sigma(\grave{\lambda})=\check{\lambda}$ in the appropriate sense.]

Apply Proposition 10 to $\left\langle P_{v \beta} ; v<\beta<\alpha\right\rangle^{*}$ in $V^{\left(B_{v}\right)}$. 
A few remarks on homogeneity will simplify some of our proofs in the next section.

A poset $P$ is homogeneous if for every $p$ and $q$ in $P_{p}$, there is an automorphism $\sigma$ of $P$ such that $\sigma(p)$ and $q$ are compatible. Every automorphism $\sigma$ of $P$ "extends" in the obvious sense to an automorphism of $B(P)$ and generates an automorphism of $V^{(B(P))}$, both of which will also be denoted by " $\sigma$ ". If $P$ is homogeneous, then the zero and the unit elements are the only elements of $B(P)$ that are invariant under all automorphisms of $B(P)$. It follows that if $\phi\left(v_{0}, \ldots, v_{n}\right)$ is a formula of ZF and $x_{0}, \ldots, x_{n}$ are elements of $V$, then $\| \phi\left(\check{x}_{0}, \ldots, \check{x}_{n} \|^{(B(P))}\right.$ is either the unit or the zero element of $B(P)$.

13. Proposition. Let $\alpha$ be an ordinal such that for all $\beta<\alpha, P_{\beta \beta+1}$ is homogeneous and definable in $V^{\left(B_{\beta}\right)}$ from elements of $\dot{V}$. Then $P_{\alpha}$ is homogeneous.

Proof. Let $a^{*}, b^{*} \in P_{\alpha}$. Define functions $a, b \in \Pi_{\beta<\alpha} P_{\beta}$ by setting $a(\beta)=\pi_{\alpha \beta}\left(a^{*}\right)$ and $b(\beta)=\pi_{\alpha \beta}\left(b^{*}\right)$ for all $\beta \leqslant \alpha$. In particular $a(\alpha)=a^{*}$ and $b(\alpha)=b^{*}$. We have to construct an automorphism $\sigma^{*}$ of $P_{\alpha}$ and an element $d^{*}$ of $P_{\alpha}$ so that $d^{*} \leqslant b^{*}$ and $d^{*} \leqslant \sigma^{*}\left(a^{*}\right)$. In fact we shall construct by induction on $\beta \leqslant \alpha$, an automorphism $\sigma_{\beta}$ of $P_{\beta}$ and an element $d_{\beta}$ of $P_{\beta}$ so that for all $\gamma \leqslant \delta \leqslant \beta$ and $p \in P_{\delta}$

(A) $\pi_{\delta \gamma}\left(\sigma_{\delta}(p)\right)=\sigma_{\gamma}\left(\pi_{\delta \gamma}(p)\right)$ and $\sigma_{\beta}\left(i_{\delta \beta}(p)\right)=i_{\delta \beta}\left(\sigma_{\delta}(p)\right)$,

(B) $d_{\beta} \leqslant{ }_{\beta} \sigma_{\beta}(a(\beta)), d_{\beta} \leqslant \beta(\beta), \pi_{\delta \gamma}\left(d_{\delta}\right)=d_{\gamma}$ and if $i_{\gamma \delta}(a(\gamma))=a(\delta)$ and $i_{\gamma \delta}(b(\gamma))=b(\delta)$, then $i_{\gamma \delta}\left(d_{\gamma}\right)=d_{\delta}$.

Case I. $\beta=0$. Let $d_{0}=0$ and let $\sigma_{0}$ be the identity restricted to $\{0\}$.

Case II. $\beta=\eta+1$. Note that $\sigma_{\eta}\left(P_{\eta \eta+1}\right)_{r}=P_{\eta \eta+1}$ because $P_{\eta \eta+1}$ is by hypothesis definable in $V^{\left(B_{\eta}\right)}$ from elements of $\dot{V}$.

If $i_{\eta \beta}(a(\eta))=a(\beta)$ and $i_{\eta \beta}(b(\eta))=b(\beta)$, let $d_{\beta}=i_{\eta \beta}\left(d_{\eta}\right)$, and set $\sigma_{\beta}\left(i_{\eta \beta}(p) q\right)=i_{\eta \beta}\left(\sigma_{\eta}(p)\right) \sigma_{\eta}(q)$ for all $p \in P_{\eta}$ and $q \in P_{\eta \eta+1}$.

Otherwise suppose that $a(\beta)=i_{\eta \beta}(a(\eta)) q_{0}$ and that $b(\beta)=i_{\eta \beta}(b(\eta)) q_{1}$, where $q_{0}, q_{1} \in \hat{P}_{\eta \eta+1}$. Since $P_{\eta \eta+1}$ is homogeneous in $V^{\left(B_{\eta}\right)}$, there is a $\tau$ that is in $V^{\left(B_{\eta}\right)}$ an automorphism of $P_{\eta \eta+1}$ such that

$$
\left\|\left(\exists q \in P_{\eta \eta+1}\right)\left(q \leqslant_{\eta \eta+1} \tau\left(\sigma_{\eta}\left(q_{0}\right)\right) \wedge q \leqslant_{\eta \eta+1} q_{1}\right)\right\|=1_{\eta} .
$$

Hence there is a $q_{2} \in \hat{P}_{\eta \eta+1}$ such that $\| q_{2} \leqslant_{\eta \eta+1} \hat{\tau}\left(q_{0}\right)$ and $q_{2} \leqslant_{\eta \eta+1} q_{1} \|=$ $1_{\eta}$. Set $d_{\beta}=i_{\eta \beta}\left(d_{\eta}\right) q_{2}$. For every $i_{\eta \beta}(p) q \in P_{\eta} \widetilde{\otimes} P_{\eta \eta+1}$ set $\sigma_{\beta}\left(i_{\eta \beta}(p) q\right)=$ $i_{\eta \beta}\left(\sigma_{\eta}(p)\right) \hat{\tau}\left(\sigma_{\eta}(q)\right)$. Then $\sigma_{\beta}$ is an automorphism of $P_{\beta}$ and the induction hypotheses hold.

Case III. $P_{\beta}$ is the inverse limit of its predecessors. For $f \in P_{\beta}$ and $\gamma<\beta$ define $d_{\beta}(\gamma)=d_{\gamma}$ and $\sigma_{\beta}(f)(\gamma)=\sigma_{\gamma}(f(\gamma)) . \sigma_{\beta}$ is an automorphism of $P_{\beta}$ and 
$d_{\beta}$ is an element of $P_{\beta}$ by the induction hypotheses.

Case IV. $P_{\beta}$ is the direct limit of its predecessors. Let $i_{\eta \beta}(p) \in P_{\beta}$ for some $p \in P_{\eta}$. Set $\sigma_{\beta}\left(i_{\eta \beta}(p)\right)=i_{\eta \beta}\left(\sigma_{\eta}(p)\right)$. $\sigma_{\beta}$ is an automorphism of $P_{\beta}$. There is an $\eta<\beta$ so that $i_{\eta \beta}(a(\eta))=a(\beta)$ and $i_{\eta \beta}(b(\eta))=b(\beta)$. Set $d_{\beta}=i_{\eta \beta}\left(d_{\eta}\right)$.

Subsection 4. Forcing with a class of conditions. Let $M=\left\langle M, M^{*} ; \in\right\rangle$ be a countable standard model of Gödel-Bernays class-set theory, where $M$ (the sets of $M$ ) and $M^{*}$ (the classes of $M$ ) are countable sets. If $B$ is in $M$ a complete Boolean algebra, $M^{(B)}$ will be the universe of $B$-sets and $B$-classes constructed in $M$. $M^{(B)}$ "satisfies" the axioms of Gödel-Bernays class-set theory.

Now suppose that the discussion of the preceding subsection took place in M. In particular, $\left\langle P_{\alpha} ; \alpha\right.$ an ordinal $\rangle$ and $\left\langle i_{\beta \alpha} ; \beta \leqslant \alpha\right\rangle$ are classes of $M$.

We now work in $M$. We leave it to the reader to translate arguments that seem to involve "classes of classes" to proper class-set theory notation.

Let $P_{\infty}$ be the direct limit of the very fine system $\left\langle P_{\alpha} ; \alpha\right.$ an ordinal $\rangle$ and $\left\langle i_{\beta \alpha} ; \beta \leqslant \alpha\right\rangle$. For every $\alpha$ let $i_{\alpha \infty}$ be the canonical embedding of $P_{\alpha}$ into $P_{\infty}$ and let $\pi_{\infty \alpha}$ be the associated projection defined so that for every $\beta$ and $p \in P_{\beta}$, $\pi_{\infty \alpha}\left(i_{\beta \infty}(p)\right)=\pi_{\beta \alpha}(p)$ if $\beta \geqslant \alpha$, and $\pi_{\infty \alpha}\left(i_{\beta}(p)\right)=i_{\beta \alpha}(p)$ if $\beta<\alpha$.

Propositions 10 to 13 extend to the case of $P_{\infty}$. For example let $v$ be an ordinal. Then there is a class $P_{v \infty}$ of $M^{\left(B_{v}\right)}$ and an isomorphism $e_{v \infty}$ of $P_{\infty}$ with $P_{v} \widetilde{\otimes} P_{v \infty}$. The latter poset is constructed using the class version of the $\widetilde{\otimes}$-operation, as is the embedding $k_{v \infty}$ of $P_{v}$ into $P_{v} \widetilde{\otimes} P_{v \infty}$. In $M^{\left(B_{v}\right)}$, the $P_{v \alpha}$ 's for $\alpha>v$ and the $j_{\beta \alpha}$ 's for $v<\beta \leqslant \alpha$ constitute a very fine system with $P_{v \infty}$ being the direct limit of the $P_{v \alpha}$ 's. If for every $\alpha \geqslant v, P_{\alpha \alpha+1}$ is $\lambda$-closed in $M^{\left(B_{\alpha}\right)}$ and if for every cardinal $\nu>v$ so that $P_{\nu}$ is the direct limit of its predecessors, $\lambda<\nu$, then $P_{v \infty}$ is $\check{\lambda}_{\text {-closed in }} M^{\left(B_{v}\right)}$ (similarly for $\lambda$-directed closure).

Let $G$ be an $M$-generic subset of $P_{\infty}$ (in addition to the usual conditions on $G$, we require that $A \cap G \neq \varnothing$ for every class $A$ of $M$ that is a dense subclass of $\left.P_{\infty}\right)$. Then $e_{v \infty}[G]$ is an M-generic subset of $P_{v} \approx P_{v \infty}, G_{v}=\left\{p \in P_{v}: i_{v \infty}(p)\right.$ $\in G\}$ is an $M$-generic subset of $P_{v}$, and $G_{v \infty}=\left\{i_{U\left(G_{v}\right)}(q): k_{v \infty}\left(1_{v}\right) q \in e_{v \infty}[G]\right\}$ is an $M\left[G_{v}\right]$-generic subset of $i_{U\left(G_{v}\right)}\left(P_{v \infty}\right)$ [cf. Proposition 6 and remarks preceding it]. Let $M[G]=\left\{x\right.$ : there is an $\alpha$ in $M$ so that $x$ is a set of $\left.M\left[G_{\alpha}\right]\right\}$.

14. Proposition. Suppose that for every ordinal $\alpha$ there is an ordinal $\eta_{\alpha} \geqslant \alpha$ so that in $\left.M^{(B} \eta_{\alpha}\right), P_{\eta_{\alpha} \infty}$ is $\stackrel{\alpha}{\alpha}$-closed. Then $M[G]$ is a model of ZFC.

Proof. Note that $M[G]$ is the directed union of transitive models of ZFC. Hence only the power set and replacement axioms are unclear. Moreover we may prove the replacement axiom in the special case when the "domain" is an ordinal.

Let $\phi\left(v_{0}, \ldots, v_{n}, v_{n+1}, v_{n+2}\right)$ be a formula of $\mathrm{ZF}$, and let $x_{0}, \ldots, x_{n}$ and $\kappa$ be elements of $M[G]$ so that $M[G] \vDash(\forall \alpha \in \kappa)(\exists ! x) \phi\left(x_{0}, \ldots, x_{n}, \alpha, x\right)$. 
Let $f$ be the function with domain $k$ so that for every $\alpha<k, M[G] \vDash$ $\phi\left(x_{0}, \ldots, x_{n}, \alpha, f(\alpha)\right)$. We will show that $f$ is in $M[G]$.

For simplicity set $v=\eta_{\kappa}, N=M\left[G_{v}\right], H=G_{v \infty}, i=i_{v\left(G_{v}\right)}$, and $P_{\infty}^{*}=$ $i\left(P_{v \infty}\right)$. For $v<\beta \leqslant \alpha$, define $j_{\beta \alpha}^{*}=i\left(j_{\beta \alpha}\right), \mu_{\alpha \beta}^{*}=i\left(\mu_{\alpha \beta}\right)$, and $P_{\alpha}^{*}=i\left(P_{v \alpha}\right)$ [cf. Proposition 11].

$P_{\infty}^{*}$ is the direct limit of the very fine system $\left.\left\langle P_{\alpha}^{*} ; \alpha\right\rangle v\right\rangle$ and $\left\langle j_{\beta \alpha}^{*} ; v<\right.$ $\beta \leqslant \alpha\rangle$. It will prove convenient (but not necessary) to assume that $P_{\infty}^{*}$ is the "union" of the $P_{\alpha}^{*}$ 's and that the $j_{\beta \alpha}^{*}$ 's are restrictions of the identity. For every $\alpha>v$ let $\mu_{\infty \alpha}^{*}$ be the projection of $P_{\infty}^{*}$ onto $P_{\alpha}^{*}$, i.e., for all $\beta>v$ and $p \in P_{P_{\beta}^{*}}$, $\mu_{\infty \alpha}^{*}(p)=\mu_{\beta \alpha}^{*}(p)$ if $\beta \geqslant \alpha$ and $\mu_{\infty \alpha}^{*}(p)=p$ if $\beta<\alpha$.

Since $P_{\infty}^{*}$ is the direct limit of the fine system of posets $\left.\left\langle P_{\alpha}^{*} ; \alpha\right\rangle v\right\rangle$, for every $\alpha>v$ the set $H_{\alpha}=H \cap P_{P_{\alpha}^{*}}$ is an N-generic subset of $P_{\alpha}^{*}$ and $\eta\left[H_{\alpha}\right]=$ $M\left[G_{\alpha}\right]$.

Define $K_{H}$ on the sets of $N$ by induction on their rank so that $K_{H}(a)=$ $\left\{K_{H}(b):(\exists p \in H)(\langle b, p\rangle \in a)\right\}$ for every $a$ a set of $N$. Let $N[H]=K_{H}[N]$, where $N$ is the class of all sets of $N$.

We now adopt (without explicitly defining) the terminology of Shoenfield's paper on unramified forcing [9] and assume that the reader is well acquainted with this paper and especially with the section on Easton forcing. We will define the forcing relation with respect to $P_{\infty}^{*}$.

Define $\Delta$ on every set $b$ of $N$ by induction on the rank of $b$ so that $\Delta(b)$ is the least $\alpha>v$ with the property that for all $a \in \operatorname{Ra}(b)$ and $p \in \operatorname{Do}(b) \cap P_{\infty}^{*}$, $\Delta(a) \leqslant \alpha$ and $p \in P_{\alpha}^{*}$. For $a$ and $b$ in $N$ set $\Delta(a, b)=\max (\Delta(a), \Delta(b))$. For every $b$ a set of $N, \Delta(b)$ is an ordinal $\alpha$ so that all the $p$ 's referred to in $b$ appear in $P_{\alpha}^{*}$. Note that for $\Delta(b) \leqslant \alpha, K_{H}(b)=K_{H_{\alpha}}(b)$, where $K_{H_{\alpha}}$ is (as in Shoenfield) defined analogously to $K_{H}$. Then for $\alpha>v, K_{H_{\alpha}}[N]=N\left[H_{\alpha}\right]=\{x: x$ is a set of $\left.N\left[H_{\alpha}\right]\right\}$ and $N[H]=\bigcup_{\alpha \in N} N\left[H_{\alpha}\right]$. It follows then that $N[H]=$ $M[G]$.

If $\phi$ is a formula of the forcing language, then " $p \mathbb{I}_{\alpha}^{*}$ " asserts that " $p$ weakly forces $\phi$ with respect to $P_{\alpha}^{*}$ ".

Now let $a, b \in N$ and $\alpha=\Delta(a, b)$ and $p \in P_{\infty}^{*}$. Define $p \mathbb{1}^{*} a \in b$ iff $\mu_{\infty \alpha}^{*}(p) \mathbb{1}_{\alpha}^{*} a \in b$, and $p \mathbb{1}^{*} a \neq b$ iff $\mu_{\infty \alpha}^{*}(p) \mathbb{1}_{\alpha}^{*} a \neq b$. [Note that $\mathbb{1}^{*}$ would be the same for any $\beta \geqslant \alpha$.]

As in Shoenfield, define $p \mathbb{1}^{*} \phi$ for $\phi$ a formula of the forcing language and $p \in P_{\infty}^{*}$ by induction on the complexity of $\phi$. Define $p \Vdash \phi$ iff $p \mathbb{H}^{*} \sim \sim \phi$. The definability, extension, and truth lemmas are proved as in Shoenfield. In the proof of these lemmas use the fact that $P_{\infty}^{*}$ is the direct limit of the fine system of the $P_{\alpha}^{*}$ 's.

Let $\mathrm{x}_{0}, \ldots, \mathrm{x}_{n}$ be names in $N$ for $x_{0}, \ldots, x_{n}$ respectively (recall that 
$M[G]=N[H])$. For $\alpha<\kappa$ let $D_{\alpha}=\left\{p \in P_{\infty}^{*}:(\exists z)(p \Vdash(\exists x \varphi(\hat{a}, x) \rightarrow\right.$ $\varphi(\hat{a}, z)))\}$. For every $\alpha<\kappa, D_{\alpha}$ is an open dense section of $P_{\infty}^{*}$. Since $P_{\infty}^{*}$ is $\kappa$-closed, $D=\bigcap_{\alpha<k} D_{\alpha}$ is also an open dense section of $P_{\infty}^{*}$. So there is a $p \in$ $H \cap D$ such that $p \Vdash(\forall \alpha \in \kappa)(\exists ! x) \phi\left(\mathrm{x}_{0}, \ldots, \mathrm{x}_{n}, \alpha, x\right)$.

For every $\alpha<k$ let $g(\alpha)$ be such that $p \Vdash \phi(\hat{\alpha}, g(\alpha))$. There is a $\delta>v$ such that $\Delta(g(\alpha))<\delta$ for all $\alpha<\kappa$. Then $f(\alpha)=K_{H}(g(\alpha))=K_{H_{\delta}}(g(\alpha))$ for all $\alpha<\kappa$. Since $g$ is in $N, f$ is in $N\left[H_{\delta}\right]$, hence in $M[G]$. It is now clear that the replacement axiom holds in $M[G]$.

A similar argument using the $\alpha$-closure of $P_{\infty}^{*}$ would show that every subset of $\kappa$ in $N[H]$ lies in $N$. Using this and the fact that the $M\left[G_{\alpha}\right]$ 's satisfy the axiom of choice, it is easy to see that the power set axiom holds in $M[G]$.

2. Applications of the Silver forcing method. Subsection two contains the main theorems of this chapter on ordinal definability. In subsection one we consider three technical constructions which will be needed to ensure that every set is ordinal definable and the G.C.H. holds in suitable Cohen extensions.

Subsection 1. Technical backward Easton constructions. The Beth function $z$ is defined on the ordinals by induction on $\alpha$ so that for $\alpha=0, z(\alpha)=\omega$; for $\alpha=\beta+1, I(\alpha)=2^{2(\beta)}$; for $\alpha$ a limit ordinal, $\supset(\alpha)=\bigcup_{\beta<\alpha} د(\beta)$. A cardinal $\nu$ is a Beth fixed point if $2(\nu)=\nu$. We use the standard interval notation for ordinals. For example, if $\alpha \leqslant \beta$, then $[\alpha, \beta)=\{\gamma: \alpha \leqslant \gamma<\beta\}$.

If $c$ is either the class of all ordinals or an ordinal greater than zero we say that $\left\langle P_{\alpha} ; \alpha \in c\right\rangle$ is a very fine sequence of posets if there is a sequence of maps $\left\langle i_{\beta \alpha} ; \beta \leqslant \alpha \in c\right\rangle$ so that $\left\langle P_{\alpha} ; \alpha \in c\right\rangle$ and $\left\langle i_{\beta \alpha} ; \beta \leqslant \alpha \in c\right\rangle$ constitute a very fine system.

15. Lemma. There is a term $C\left(v_{0}, v_{1}\right)$ of $\mathrm{ZF}$ so that if $\nu<\lambda$ are Beth fixed points, then $C(\nu, \lambda)$ is a v-directed closed poset with the property that $V^{(B(C(\nu, \lambda)))} \vDash(\hat{v}$ and $\grave{\lambda}$ are Beth fixed points and the G.C.H. holds in the interval $[\check{\nu}, \check{\lambda}))$.

PROOF. Let $\theta\left(v_{0}\right)$ be a term of ZF so that for every cardinal $k$ if $2^{k}>k^{+}$ then $\theta(\kappa)$ is the poset $R$ with $P_{R}=\{p: p$ is a function with domain a subset of $\kappa+$ and range a subset of $2^{\kappa}$ so that $\left.|p| \leqslant k\right\}$ and for $p, q \in P_{R}, p<{ }_{R} q$ if $p 2$ $q$; if $2^{K}=k^{+}$, then $\theta(\kappa)=\langle\{0\} ;=\rangle$. In any case $\theta(\kappa)$ is a homogeneous, $k$-directed closed poset and $V^{(B(\theta(\kappa)))} \vDash 2^{\check{\kappa}}=(\check{\kappa})^{+}$.

Let $\nu<\lambda$ be Beth fixed points. Define by induction on $\alpha<\lambda$ a very fine sequence of posets $\left\langle P_{\alpha} ; \alpha\langle\lambda\rangle\right.$ and a strictly increasing sequence of cardinals $\left\langle\nu_{\alpha} ; \alpha\langle\lambda\rangle\right.$ so that for every $\alpha\left\langle\lambda, P_{\alpha}\right.$ is a homogeneous, $\nu$-directed closed poset and $\left|P_{\alpha}\right|$ and $\nu_{\alpha}$ are less than $z(\nu+\alpha+\omega)$. The verification of the cardinality estimates is left to the reader. Homogeneity follows from Proposition 13. 
Case I. For $\alpha=0$ let $\nu_{0}=\nu$ and $P_{0}=\langle\{0\} ;=\rangle$.

Case II. For $\alpha=\beta+1$ let $V^{\left(B_{\beta}\right)}=P_{\beta \alpha}=\theta\left(\check{\nu}_{\beta}\right)$. Set $P_{\alpha}=P_{\beta} \widetilde{\otimes} P_{\beta \alpha}$. Define $\nu_{\alpha}$ to be the unique cardinal in $[\nu, \lambda)$ so that $V^{\left(B_{\alpha}\right)} \vDash\left(\check{\nu}_{\alpha}\right.$ is the least cardinal greater than $\check{\nu}_{\beta}$ ).

Case III. For $\alpha$ an inaccessible cardinal $>\nu$ let $P_{\alpha}$ be the direct limit of its predecessors and set $\nu_{\alpha}=\bigcup_{\beta<\alpha} \nu_{\beta}$. By the induction hypotheses $\nu_{\alpha}=\alpha$.

Case IV. For $\alpha$ a limit ordinal that is not an inaccessible cardinal $>v$ let $P_{\alpha}$ be the inverse limit of its predecessors and set $\nu_{\alpha}=\bigcup_{\beta<\alpha} \nu_{\beta}$. Set $C(\nu, \lambda)=$ $P_{\lambda}$, the inverse limit of the $P_{\alpha}$ 's.

We first show that for every $\alpha<\lambda, V^{\left(B_{\lambda}\right)} \vDash\left(\check{\nu}_{\alpha+1}=\left(\check{\nu}_{\alpha}\right)^{+}\right)$. By the construction $V^{\left(B_{\alpha+1}\right)} \vDash\left(\check{\nu}_{\alpha+1}=\left(\check{\nu}_{\alpha}\right)^{+}\right)$. By Proposition 11 there is a poset $P_{\alpha+1 \lambda}$ in $V^{\left(B_{\alpha+1}\right)}$ such that $P_{\lambda}$ is isomorphic to $P_{\alpha+1} \widetilde{\otimes} P_{\alpha+1 \lambda}$ and $V^{\left(B_{\alpha+1}\right)} F$ $\left(P_{\alpha+1 \lambda}\right.$ is $\check{\nu}_{\alpha+1}$-closed $)$. Then $V^{\left(B_{\alpha+1}\right)} \vDash\left(V^{\left(B\left(P_{\alpha+1 \lambda}\right)\right)} \vDash\left(\left(\check{\nu}_{\alpha+1}\right)^{\gamma}=\right.\right.$ $\left.\left.\left(\left(\tilde{\nu}_{\alpha}\right)^{r}\right)^{+}\right)\right)$. Since $V^{\left(B_{\lambda}\right)}$ is "isomorphic" to the $B\left(P_{\alpha+1 \lambda}\right)^{\sim}$-relation system whose underlying set is $\left\{x \in V^{\left(B_{\alpha+1}\right)}:\left\|x \in V^{\left(B\left(P_{\alpha+1 \lambda}\right)\right)}\right\|=1_{B_{\alpha+1}}\right\}$ [cf. proof of Case II of Proposition 11], $V^{\left(B_{\lambda}\right)} \vDash\left(\check{\nu}_{\alpha+1}=\left(\check{\nu}_{\alpha}\right)^{+}\right)$.

Now suppose that $\|$ The G.C.H. does not hold in $[\check{\nu}, \check{\lambda}) \|^{\left(B_{\lambda}\right)} \neq 0_{B_{\lambda}}$. By the homogeneity of $B_{\lambda}$ [cf. Proposition 13] there is an $x \in V^{\left(B_{\lambda}\right)}$ so that $\| x$ is the least cardinal in $\left[\grave{\nu}, \grave{\lambda}\right.$ ) such that $2^{x} \neq x^{+} \|=1_{B_{\lambda}}$. But by the preceding paragraph and the homogeneity of $B_{\lambda}$ there is an $\alpha\left\langle\lambda\right.$ such that $\left\|x=\check{\nu}_{\alpha}\right\|=1_{B_{\lambda}}$. Now use the closure conditions, the essential property of $\theta\left(\nu_{0}\right)$, and an argument similar to that of the preceding paragraph to get a contradiction.

16. Construction. Let $\nu<\lambda$ be Beth fixed points so that the G.C.H. holds in $[\nu, \lambda)$. Let $A=\{\gamma \in[\nu, \lambda): \gamma$ is a regular cardinal $\}$. Suppose that $f$ : $A \rightarrow[\nu, \lambda)$ is so that

(a) For every $\gamma \in A, f(\gamma)$ is a cardinal with cofinality $>\gamma$.

(b) For all $\gamma \leqslant \delta$ in $A, f(\gamma) \leqslant f(\delta)$.

For every $\delta \in A$ let $Q_{\delta}=\{\langle\gamma, \alpha, \beta\rangle: \delta \geqslant \gamma \in A, \alpha<f(\gamma)$, and $\beta<\gamma\}$. Let $Q=\bigcup_{\delta \in A} Q_{\delta}$. Set $P_{R}=\{p: p$ is a function, domain $(p) \subseteq Q$, range $(p)$ $\subseteq 2$, and for all $\gamma \in A \mid$ domain $\left.(p) \cap Q_{\gamma} \mid<\gamma\right\}$. For $p$ and $q$ in $P_{p}$, set $p \leqslant_{R} q$ if $p \supseteq q$. There is a term $E\left(v_{0}\right)$ of ZF so that for every function $f$ with the above properties $E(f)$ is the poset $R=\left\langle P_{R} ;\langle R\rangle\right.$. For every $\alpha\langle\nu, E(f)$ is $\alpha$-directed closed. The paper of Shoenfield $(E(f)$ is essentially due to Easton [1]) on unramified forcing [9] shows that if the G.C.H. holds in $[\nu, \lambda]$, then every $\gamma \in A$, $\gamma$ is a regular cardinal and $2^{\gamma}=f(\gamma)^{\gamma}$ in $V^{(B(E(f)))}$.

The remaining material in subsection 1 is relevent only to Theorems 20 and 21; the reader interested in Theorem 18 may skip directly there.

Henceforth $\omega\left(v_{0}\right)$ will be a term of ZF enumerating in increasing order the class of all infinite cardinals. 
Before proceeding with the next lemma we recall two constructions involving Kurepa trees. We use (without explicitly defining) the terminology of Jech's article on trees [4].

Let $\alpha$ be an ordinal. We shall define the poset $R$ of Stewart's conditions for adding a Kurepa tree on $\omega(\alpha+1)$ [13].

$P_{R}$ is the set of all ordered pairs $\langle L, f\rangle$ with the following properties:

(i) $L=\langle T$; $\leqslant\rangle$ is a tree.

(ii) $T \subseteq \omega(\alpha+1)$ so that $|T|<\omega(\alpha+1)$.

(iii) $f$ is an injective function with domain a subset of $\omega(\alpha+2)$ of cardinality less than $\omega(\alpha+1)$. The range of $f$ is a subset of the set of all branches of $L$ of length $\beta$, where $\beta$ is the length of $L$.

(iv) For every point $x$ in $T$ there is a branch $b$ in the range of $f$ so that $x \in b$.

For $\left\langle L_{0}, f_{0}\right\rangle$ and $\left\langle L_{1}, f_{1}\right\rangle$ in $P_{R},\left\langle L_{0}, f_{0}\right\rangle \leqslant_{R}\left\langle L_{1}, f_{1}\right\rangle$ if $L_{0}$ is an endextension of $L_{1}$, if domain $\left(f_{0}\right) \supseteq\left(f_{1}\right)$, and if $f_{0}(\beta) \supseteq f_{1}(\beta)$ for all $\beta$ in the domain of $f_{1}$.

Let $\sigma\left(v_{0}\right)$ be a term of ZF so that if $\alpha$ is an ordinal, then $\sigma(\alpha)$ is the poset $R$ of Stewart's conditions defined above. $\sigma(\alpha)$ is an $\omega(\alpha)$-directed closed poset so that if the G.C.H. holds, then the cardinals of $V$ are cardinals in $V^{(B(\sigma(\alpha)))}$, and the G.C.H. holds and there is a Kurepa tree on $\omega(\alpha+1)^{\gamma}$ in $V^{(B(\sigma(\alpha)))}$. [The proof of [4] is only for $\alpha=0$. But it extends without serious difficulties to the more general context.]

Now let $\alpha$ be an ordinal so that there is an inaccessible cardinal greater than $\omega(\alpha)$. We shall describe Levy's condition $R$ for forcing the least inaccessible cardinal $\kappa>\omega(\alpha)$ to be $\omega(\alpha+2)$ in the Cohen extension.

$P_{R}=\{f \subseteq \kappa \times \omega(\alpha+1) \times \kappa: f$ is a function, $|f|<\omega(\alpha+1)$, and for all $(\gamma, \delta) \in$ domain $(f), f(\gamma, \delta) \in \omega(\gamma)\}$. For $f_{0}$ and $f_{1}$ in $P_{R}, f_{0} \leqslant_{R} f_{1}$ if $f_{0} \supseteq f_{1}$.

There is a term $\tau\left(v_{0}\right)$ of ZF so that if $\alpha$ is an ordinal with the property that there is an inaccessible cardinal greater than $\omega(\alpha)$, then $\tau(\alpha)$ is the poset $R$ defined above. In this case, $\tau(\alpha)$ is $\omega(\alpha)$-directed closed. Silver has shown [10] that if the G.C.H. holds, then in $V^{(B(\tau(\alpha)))}$, the G.C.H. holds and there are no Kurepa trees on $\omega(\alpha+1)^{\gamma}$.

17. Lemma. Let $\nu<\lambda$ be Beth fixed points so that $\mid\{\alpha \in[\nu, \lambda): \alpha$ is an inaccessible cardinal $\} \mid \geqslant \nu$ and $(\forall$ cardinal $\kappa<\lambda)\left(2^{\kappa}=\kappa^{+}\right)$. Let $A$ be $a$ subset of $\nu$, and let $\eta: \nu \rightarrow \nu$ enumerate in increasing order the set of all limit ordinals less than $\nu$. There is a term $\chi\left(v_{0}, v_{1}\right)$ of ZF so that for $A$ and $\nu$ as above, $\chi(\nu, A)$ is a v-directed closed poset and for every $\gamma<\nu$, \|There is a Kurepa tree on $\omega\left((\nu+\eta(\gamma)+1)^{\gamma}\right) \|=1_{B(\chi(\nu, A))}$ if $\gamma \in A$ and $\|$ There is $a$ Kurepa tree on 
$\omega\left((\nu+\eta(\gamma)+1)^{\nu}\right) \|=0_{B(\chi(\nu, A))}$ if $\gamma \notin$.A. Also the G.C.H. holds beneath $\lambda^{\nu}$ in $V^{(B(\chi(\nu, A)))}$.

Proof. Define by induction on $\gamma \leqslant \nu$ a very fine sequence of posets $\left(P_{\gamma} ; \gamma \leqslant \nu\right)$ as follows.

Case I. For $\gamma=0$, let $P_{0}=\langle\{0\} ;=\rangle$.

Case II. For $\gamma=\delta+1$, let

$$
V^{(B \delta)} \vDash P_{\delta \gamma}= \begin{cases}\sigma\left((\nu+\eta(\delta))^{\nu}\right) & \text { if } \check{\delta} \in \check{A}, \\ \tau\left((\nu+\eta(\delta))^{\nu}\right) & \text { if } \check{\delta} \notin \check{A} .\end{cases}
$$

Case III. For $\gamma$ a limit ordinal, let $P_{\gamma}$ be the inverse limit of its predecessors. Set $\chi(\nu, A)=P_{\nu}$.

We shall make a few remarks on the proof but shall leave the detailed verifications to the reader.

Roughly, the argument needed to establish that the G.C.H. holds beneath $\lambda$ in $V^{\left(B_{\nu}\right)}$ proceeds by induction on $\gamma \leqslant \nu$ and uses the relevant closure conditions on the $P_{\gamma \nu}$ 's [cf. proof of Lemma 15], the usual cardinality arguments on the $P_{\gamma}$ 's, and the fact that the forcing conditions $\sigma\left(\nu_{0}\right)$ and $\tau\left(v_{0}\right)$ preserve the G.C.H. The only difficulty arises when $\gamma$ is a limit ordinal. For this case note that in $V^{\left(B_{\nu}\right)}, \kappa=\omega\left((\nu+\eta(\gamma))^{r}\right)$ is a strong limit cardinal of cofinality $\leqslant \check{\gamma}$ and hence $2^{\kappa}=k^{\gamma}$. Then use the fact that $P_{\nu}$ is $\gamma$-closed.

To complete the outline-proof, fix $\gamma<\nu$. If $\gamma \in A$, then $V^{\left(B_{\gamma+1}\right)} \vDash$ (There is a Kurepa tree on $\omega\left((\nu+\eta(\gamma)+1)^{\gamma}\right)$ and $P_{\gamma+1 \nu}$ is $\omega\left((\nu+\eta(\gamma)+3)^{\gamma}\right)$ closed). As in the proof of Lemma 15, it follows that in $V^{\left(B_{\nu}\right)}$, there is a Kurepa tree on $\omega\left((\nu+\eta(\gamma)+1)^{r}\right)$. The proof is similar for $\gamma \notin A$.

Subsection 2. The main theorems. The following result is a generalization of the main theorem of Easton [1]. The new element in our result is that the property of supercompactness is preserved.

18. THEOREM. Let $M=\left\langle M, M^{*} ; \in\right\rangle$ be a countable standard model of Gödel-Bernays class-set theory so that the G.C.H. holds in M. Let I be a classfunction of $M$ from the regular cardinals of $M$ into the cardinals of $M$ so that for all regular cardinals $\nu \leqslant \lambda$ in $M$

(1) cofinality $(I(\nu))>\nu$ and

(2) $I(\nu) \leqslant I(\lambda)$

Assume in addition that there is a statement $\psi$ and a formula $\phi\left(v_{0}, v_{1}\right)$ of $\mathrm{ZF}$ so that $\psi$ is true in $M$ and so that $M \vDash((\forall$ cardinal $\gamma)(R(\gamma) \vDash \psi \rightarrow I[\gamma] \subseteq \gamma$ and $(\forall \alpha, \beta \in \gamma)(I(\alpha)=\beta \leftrightarrow R(\gamma) \vDash \phi(\alpha, \beta))))$. [This condition states that in $M, I$ is $\Delta_{2}$ in the Levy hierarchy.]

There is a class of conditions $P_{\infty}$ in $M$ so that if $N$ is any Cohen extension 
of $M$ with respect to $P_{\infty}$ then

(1) $N$ is a model of ZFC.

(2) $M$ and $N$ have the same regular cardinals.

(3) For every regular cardinal $\nu$ in $N, I(\nu)=2^{\nu}$ in $N$.

(4) Every supercompact cardinal in $M$ is supercompact in $N$.

Proof. Conditions (1) and (2) on $I$ are the usual Easton requirements. The additional condition on $I$ is a local definability requirement needed to show that supercompactness is preserved in Cohen extensions of $M$ with respect to $P_{\infty}$.

We work in $M$. Let $e$ be a class-function enumerating in increasing order the closed unbounded class $F=\left\{\alpha: \alpha\right.$ is a limit point of the set $\left\{\beta: \beta=\beth_{\beta}\right.$ and $R(\beta) \vDash \psi\}$. For $\alpha<\beta$ in $F$ let $I_{\alpha \beta}=I \uparrow[\alpha, \beta)$ and set $I_{\alpha}=I \uparrow \alpha$. Note that for every $\alpha$ in $F, \alpha=\beth_{\alpha}$ and $I_{\alpha}$ is definable in $R(\alpha)$, that is

$$
\text { (A) }(\forall \gamma<\alpha)(\forall \delta)\left(I_{\alpha}(\gamma)=\delta \leftrightarrow R(\alpha) \vDash(\exists \gamma)(R(\gamma) \vDash \psi \wedge \phi(\gamma, \delta))\right) \text {. }
$$

Define by induction on the ordinal $\alpha$ a very fine class-sequence of posets $\left\langle P_{\alpha} ; \alpha\right.$ an ordinal $\rangle$ so that for every regular cardinal $\nu, M^{\left(B_{\alpha}\right)} \vDash(\tilde{v}$ is a regular cardinal) and so that in $M^{\left(B_{\alpha}\right)}$, the G.C.H. holds for all cardinals $\lambda \geqslant e(\alpha)^{r}$.

Case I. For $\alpha=0$, let $P_{0}=\langle\{0\} ;=\rangle$.

Case II. For $\alpha=\beta+1$, let $M^{\left(B_{\beta}\right)} \vDash\left(P_{\beta \alpha}=E\left(I_{e(\beta) e(\alpha)}\right)\right)$ [cf. Construction 16], and set $P_{\alpha}=P_{\beta} \widetilde{\otimes} P_{\beta \alpha}$.

Case III. For $\alpha$ an inaccessible cardinal so that $(\forall \beta<\alpha)\left(M^{\left(B_{\beta}\right)} \vDash\left|P_{\beta \beta+1}\right|\right.$ $<\stackrel{\alpha}{\alpha}$ ), note that $e(\alpha)=\alpha$ and let $P_{\alpha}$ be the direct limit of its predecessors.

Case IV. If $\alpha$ is a limit ordinal and Case III does not hold, let $P_{\alpha}$ be the inverse limit of its predecessors.

Let $P_{\infty}$ be the direct limit of the $P_{\alpha}$ 's and let $G_{\infty}$ be an $M$-generic subclass of $P_{\infty}$. Set $N=M\left[G_{\infty}\right]=\left\{x\right.$ : there is an $\alpha$ in $M$ so that $x$ is a set of $\left.M\left[G_{\alpha}\right]\right\}$ [cf. remarks preceding Proposition 14].

By Corollary 12 and Proposition $14, N$ is a model of ZFC.

Note that for every $\alpha$ in $M, N$ is a Cohen extension of $M\left[G_{\alpha}\right]$ by means of a poset which is $\gamma$-directed closed for every $\gamma<e(\alpha)$. By this remark and by Construction 16 and routine arguments, $N$ and $M$ have the same regular cardinals and for every regular cardinal $\nu$ in $N, N \vDash\left(2^{\nu}=I(\nu)\right)$.

It remains to show that every supercompact cardinal in $M$ is supercompact in $N$. For this we shall need a local definability property of the $P_{\alpha}$ 's and a general observation regarding Cohen extensions.

FACT B. There is a term $v\left(v_{0}\right)$ of ZF so that in $M, v(\alpha)=P_{\alpha}$ for every ordinal $\alpha$, and for every fixed point $\alpha$ of $e$ and $\beta<\alpha, R(\alpha) \vDash v(\beta)=P_{\beta}$.

Fact $B$ follows readily from Fact $A$ and from an inspection of the definition of the $P_{\alpha}$ 's. 
REMARK C. Let $M^{*} \subseteq M$ be countable standard models of ZFC with the same ordinals so that $R(\alpha) \cap M^{*} \in M$ for every $\alpha$ in $M$. Suppose that $\lambda$ is a cardinal in $M$ so that ${ }^{\lambda} M^{*} \cap M \subseteq M^{*}$ and that in $M^{*}, P$ is a poset with $\left|P_{p}\right| \leqslant \lambda$. Let $G$ be an $M$-generic subset of $P$. Then ${ }^{\lambda} M^{*}[G] \cap M[G] \subseteq M^{*}[G]$.

Proof of RemarK. Let $f \in{ }^{\lambda} M^{*}[G] \cap M[G]$ and $\mathrm{f} \in M$ so that $K_{G}$ (f) $=f$ [we use Shoenfield's notation [9]]. There is a $d \in M^{*}$ and a $p_{0} \in G$ so that in $M, p_{0} \Vdash \mathbf{f}: \hat{\lambda} \rightarrow d$. Let $d^{*}$ be the intersection of all transitive sets $b$ so that $d \in b$. Then $d^{*} \in M^{*}$.

For every $\alpha<\lambda$ let $A_{\alpha}=\left\{p \leqslant_{p} p_{0}:\left(\exists x \in d^{*}\right)(p \Vdash((\exists y)(\mathrm{f}(\hat{\alpha})=y)\right.$ $\rightarrow y=x)$ )\}. $A_{\alpha}$ is a dense subset of $P$ beneath $p_{0}$. Let $g$ be a function so that domain $(g)=\left\{\langle\alpha, p\rangle: \alpha\left\langle\lambda\right.\right.$ and $\left.p \in A_{\alpha}\right\}$ and so that for $\langle\alpha, p\rangle$ in the domain of $g, p \Vdash((\exists y)(f(\hat{\alpha})=y) \rightarrow y=g(\hat{\alpha}, p))$ and $g(\alpha, p) \in d^{*}$. By our assumptions on $M, M^{*}$, and $P, g \in M^{*}$. Routine arguments show that for every $\alpha<\lambda, A_{\alpha} \cap G \neq \varnothing$ and for every $p \in A_{\alpha} \cap G, K_{G}(g(\alpha, p))=f(\alpha)$. Then $f \in$ $M^{*}[G]$.

Now suppose that $k$ is supercompact in $M$.

We first show that $k$ is in $F$. It will then follow by the inaccessibility of $k$ that in fact $e(k)=k$. We work in $M$. Let $\beta>k$ be so that $د_{\beta}=\beta$ and $R(\beta) \vDash$ $\psi$. Let $\mu$ be a normal measure on $p_{\kappa} \beta$ and $j: M \rightarrow j(M) \simeq V^{p_{\kappa} \beta} / \mu$ the associated embedding. By the remarks in $\S 0, j(M)$ is closed under $\beta$-sequences and $j(k)>\beta$. Since $د_{\beta}=\beta$, it follows that $R(\beta) \in j(M)$ and that $j(M) \vDash\left(R(\beta) \vDash \psi\right.$ and $\beth_{\beta}=$ $\beta)$. So if ${ }^{\top}\left\langle\beta_{x}: x \in P_{k} \beta\right\rangle^{\urcorner}=\beta$, then $R\left(\beta_{x}\right) \vDash \psi, \beth_{\beta_{x}}=\beta_{x}$, and $\beta_{x}<k$ a.e. with respect to $\mu$. Now note that by the $\kappa$-additivity of $\mu, \kappa$ is the least ordinal so that $\beta_{x}<\kappa$ a.e. with respect to $\mu$. It follows that $\kappa$ is in $F$.

LEMMA. $\boldsymbol{K}$ is supercompact in $N$.

Proof. Our proof is fairly general and depends only on the closure properties of the $P_{\alpha}$ 's and on Fact $B$.

Let $k \leqslant \nu^{\prime}<\nu<\lambda<\lambda^{\prime}$ be fixed points of $e$.

Working in $M$ let $\mu$ be a normal measure on $p_{\kappa} \lambda^{\prime}$ and $j$ the associated elementary embedding of $M$ into $j(M) \simeq M^{p_{\kappa} \lambda^{\prime}} / \mu$. By the discussion in $\S 0$, we have that $\lambda^{\prime} j(M) \subseteq j(M), R\left(\lambda^{\prime}\right) \in J(M)$, and $j(k)>\lambda^{\prime}$. Let $\mu^{\prime}$ be the projection of $\mu$ on $p_{k} \nu^{\prime}$. Recall that $\mu^{\prime}$ is a normal measure on $p_{k} \nu^{\prime}$ so that for every $A \subseteq p_{k} \nu^{\prime}$, $\mu^{\prime}(A)=1$ iff $j\left[\nu^{\prime}\right] \in j(A)$.

To facilitate the understanding of the proof we first give a preview. Since in $M, P$ is a limit of a very fine sequence of posets, in $j(M), j\left(P_{\nu}\right)$ is also a limit of a very fine sequence of posets. Now the $P_{\alpha}$ 's are "locally definable", and $M$ and $j(M)$ have the same $\lambda^{\prime}$-sequences. It follows by an application of Propositions 11 and 12 to $j\left(P_{\nu}\right)$ in $j(M)$, that in the terminology of these propositions, $j(M)$ $\vDash\left[\right.$ There is a $P_{\lambda j(\nu)}^{*} \in j(M)^{\left(B_{\lambda}\right)}$ with an isomorphism $d: j\left(P_{\nu}\right) \rightarrow P_{\lambda} \widetilde{\otimes} P_{\lambda j(\nu)}^{*}$ 
and $j(M)^{\left(B_{\lambda}\right)} \vDash\left(P_{\lambda j(\nu)}^{*}\right.$ is $\gamma$-directed closed for every $\left.\left.\gamma<\lambda^{\nu}\right)\right]$.

Now let $i$ be the interpretation of $j(M)^{\left(B_{\lambda}\right)}$ with respect to $U\left(G_{\lambda}\right)$ [cf. discussion preceding 6]. Since in $j(M)\left[G_{\lambda}\right], i\left(P_{\lambda j(\nu)}^{*}\right)$ is a $\gamma$-directed closed poset for every $\gamma<\lambda$, it is also the case that $i\left(P_{\lambda j(\nu)}^{*}\right)$ is a $\gamma$-directed closed poset for every $\gamma<\lambda$ in $M$.

Using this closure property of $i\left(P_{\lambda j(v)}^{*}\right)$, we shall choose, in a manner to be described below, an $M\left[G_{\lambda}\right]$-generic subset $H$ of $i\left(P_{\lambda j(\nu)}^{*}\right)$ so that if $H^{*}=$ $d^{-1}\left[G_{\lambda} \widetilde{\otimes} H\right] \subseteq j\left(P_{\nu}\right)$, then $j\left[G_{\nu}\right] \subseteq H^{*}$. This will allow us to define in $M\left[G_{\lambda}\right]$ - $[H]$ an elementary embedding $k: M\left[G_{\nu}\right] \rightarrow j(M)\left[H^{*}\right]$ extending the embedding $j: M \rightarrow j(M)$ as follows:

$$
k\left(K_{G_{\nu}}(x)\right)=K_{H^{*}}(j(x)) .
$$

In verifying that $(*)$ gives a well-defined map we shall need the fact that for every $p \in G_{\nu}, j(p) \in H^{*}$. To establish this fact we shall use an important idea of Silver to show the existence of a single master condition $q \in P_{\lambda j(\nu)}^{*}$ so that if $H$ is any $\underset{\sim}{M}\left[G_{\lambda}\right]$-generic subset of $i\left(P_{\lambda j(\nu)}^{*}\right)$ with $i(q) \in H$, then $j\left[G_{\nu}\right] \subseteq H^{*}=$ $d^{-1}\left[G_{\lambda} \widetilde{\otimes} H\right]$. Silver uses a variant of this idea to prove the consistency of the failure of the G.C.H. at a measurable cardinal.

The embedding $k$ does not lie in $M\left[G_{\nu}\right]$ : since $M\left[G_{\nu}\right] \vDash\left(G_{\nu}\right.$ is an $M$-generic subset of $\left.P_{\nu}\right)$, then $k\left(M\left[G_{\nu}\right]\right) \vDash\left(k\left(G_{\nu}\right)\right.$ is a $k(M)$-generic subset of $\left.k\left(P_{\nu}\right)\right)$. But $k\left(P_{\nu}\right)=j\left(P_{\nu}\right) \simeq P_{\lambda} \widetilde{\otimes} P_{\lambda j(v)}^{*}$.

Finally we shall define by means of $k$ a set $\mu^{*}$ so that in $M\left[G_{\nu}\right], \mu^{*}$ is a normal measure on $p_{\kappa} \nu^{\prime}$ extending $\mu^{\prime}$. Since $\mu^{*}$ is definable from $k$ and $k$ lies in $M\left[G_{\lambda}\right][H], \mu^{*}$ is also in $M\left[G_{\lambda}\right][H]$. But then $\mu^{*}$ is already in $M\left[G_{\lambda}\right]$ because $M\left[G_{\lambda}\right][H]$ and $M\left[G_{\lambda}\right]$ have the same $\nu$-sequences.

We now proceed with the proof. Since in $M,\langle v(\alpha) ; \alpha \leqslant \nu\rangle$ is a very fine sequence of posets, it follows that $j(M) \vDash(\langle v(\alpha) ; \alpha \leqslant j(\nu)\rangle$ is a very fine sequence of posets). For $\beta \leqslant \alpha \leqslant j(\nu)$ in $j(M)$, let $v(\alpha)=P_{\alpha}^{*}$ and let $i_{\beta \alpha}^{*}$ be the canonical embedding of $B\left(P_{\beta}^{*}\right)$ and $\pi_{\alpha \beta}^{*}$ the associated projection. Since $R(\lambda) \in j(M)$, Fact B shows that for $\beta \leqslant \alpha \leqslant \lambda, P_{\alpha}^{*}=P_{\alpha}, i_{\beta \alpha}^{*}=i_{\beta \alpha}$ and $\pi_{\alpha \beta}^{*}=\pi_{\alpha \beta}$. Note that $j\left(P_{\nu}\right)=P_{j(\nu)}^{*}$ In $j(M)$ there is an isomorphism $d: P_{j(\nu)}^{*} \rightarrow P_{\lambda} \widetilde{\otimes} P_{\lambda j(\nu)}^{*}$, where in $j(M)^{\left(B_{\lambda}\right)}, P_{\lambda j(\nu)}^{*}$ is $\gamma$-directed closed for every $\gamma<e(\lambda)^{\nu}$.

In $j(M)$ let $f=i_{B_{\lambda}} B\left(P_{\lambda j(\nu)}^{*}\right)$ [cf. remark preceding 1], and let $i$ be the interpretation of $j(M)^{\left(B_{\lambda}\right)}$ with respect to the $M$-complete ultrafilter $U\left(G_{\lambda}\right)$ on $B_{\lambda}$. Note that if $f(p) q \in P_{\lambda} \widetilde{\otimes} p_{\lambda j(\nu)}^{*}$ and $p \in G_{\lambda}$, then $i(f(p) q)=i(q)$.

Fix $s \in P_{\nu}$. Since $P_{\kappa}$ is the direct limit of its predecessors, there is an $\alpha<$ $\kappa$ and an $s_{0} \in P_{\alpha}$ so that $\pi_{\nu \kappa}(s)=i_{\alpha \kappa}\left(s_{0}\right)$. Since $P_{\alpha} \subseteq R(\kappa), j\left(s_{0}\right)=s_{0}$ and so $\pi_{j(\nu) j(\kappa)}^{*}(j(s))=i_{\alpha j(\kappa)}^{*}\left(s_{0}\right)$. [To ensure that this be true is the only reason for 
taking the direct limit at certain limit stages of the construction.] It follows that $d(j(s))=f\left(i_{\alpha \lambda}\left(s_{0}\right)\right) s_{1}$ for some $s_{1} \in P_{\lambda j(\nu)}^{*}$. If in addition, $s \in G_{\nu}$, then $i_{\alpha \lambda}\left(s_{0}\right) \in G_{\lambda}$ and $i(d(j(s)))=i\left(s_{1}\right)$.

Now note that $j \uparrow P_{\nu}$ is in $j(M)$. In $j(M)\left[G_{\lambda}\right]$ define $A=\{i(d(j(s))): s \in$ $G_{\nu}$ \}. From the preceding paragraph and the fact that $G_{\nu}$ is a directed subset of $P_{\nu}$, it follows that in $j(M)\left[G_{\lambda}\right], A$ is a directed subset of $i\left(P_{\lambda j(\nu)}^{*}\right)$ of cardinality less than $\lambda$. Hence there is a $q \in P_{\lambda j(v)}^{*}$ so that $i(q) \leqslant a$ for every $a \in A$ in the poset ordering of $i\left(P_{\lambda j(v)}^{*}\right)$.

Let $H$ be an $M\left[G_{\lambda}\right]$-generic subset of $i\left(P_{\lambda j(\nu)}^{*}\right)$ so that $i(q) \in H$. Then $G_{\lambda}$ $\widetilde{\otimes} H=\left\{f(p) q: p \in G_{\lambda}\right.$ and $\left.i(q) \in H\right\}$ is a $j(M)$-generic subset of $P_{\lambda} \widetilde{\otimes} P_{\lambda j(v)}^{*}$ so that $j\left[G_{\nu}\right] \subseteq d^{-1}\left[G_{\lambda} \widetilde{\otimes} H\right]=H^{*}$.

By Remark $\mathrm{C}, \lambda_{j}(M)\left[G_{\lambda}\right] \cap M\left[G_{\lambda}\right] \subseteq j(M)\left[G_{\lambda}\right]$. Hence $i\left(P_{\lambda j(\nu)}^{*}\right)$ is $\gamma$-closed in $M\left[G_{\lambda}\right]$ for every $\gamma<\lambda$. Then $R(\nu) \cap M\left[G_{\nu}\right]=R(\nu) \cap M\left[G_{\lambda}\right]=$ $R(\nu) \cap M\left[G_{\lambda}\right][H]$.

Define in $M\left[G_{\lambda}\right][H]$, an elementary embedding $k: M\left[G_{\nu}\right] \rightarrow j(M)\left[H^{*}\right]$ so that $k\left(K_{G_{\nu}}(x)\right)=K_{H^{*}}(j(x))$ for every $x \in M$. It is easy to see that $k$ is a well-defined elementary embedding. For example let $\phi\left(v_{0}, \ldots, v_{n}\right)$ be a formula of $\mathrm{ZF}$ and $x_{0}, \ldots, x_{n} \in M\left[G_{\nu}\right]$ so that $M\left[G_{\nu}\right] \vDash \phi\left(x_{0}, \ldots, x_{n}\right)$. There is a $p \in G_{\nu}$ so that $p \|_{P_{\nu}} \phi\left(\mathrm{x}_{0}, \ldots, \mathrm{x}_{n}\right)$ where $K_{G_{\nu}}\left(\mathrm{x}_{m}\right)=x_{m}$ for all $m \leqslant n$. Then in $j(M), j(p) \underset{j\left(\mathrm{P}_{\nu}\right)}{\Vdash} \phi\left(j\left(\mathrm{x}_{0}\right), \ldots, j\left(\mathrm{x}_{n}\right)\right)$ and $j(p) \in H^{*}$. It follows that $j(M)\left[H^{*}\right] \vDash \phi\left(k\left(x_{0}\right), \ldots, k\left(x_{n}\right)\right)$.

We show that $k$ extends $j$. For every poset $P$ let ${ }^{\wedge} P$ be the operation defined on every set $x \in M$ by induction on the rank of $x$ so that $\hat{x}^{p}=\left\{\left\langle p, \hat{y}^{p}\right\rangle\right.$ : $y \in x$ and $\left.p \in P_{P}\right\}$. Then if $G$ is any $M$-generic subset of $P, K_{G}\left(\hat{x}^{P}\right)=x$ for every $x \in M$. In particular, for every $x \in M$,

$$
k(x)=k\left(K_{G_{\nu}}\left(\check{x}^{P_{\nu}}\right)\right)=K_{H} *\left(j\left(\hat{x}^{P_{\nu}}\right)\right)=K_{H^{*}}\left(j(x)^{\wedge} j\left(P_{\nu}\right)=j(x) .\right.
$$

Define a measure $\mu^{*}$ on the subsets $A$ of $p_{\kappa} \nu^{\prime}$ in $M\left[G_{\nu}\right]$ so that $\mu^{*}(A)=1$ iff $j\left[\nu^{\prime}\right] \in k(A)$. Clearly $\mu^{*}$ lies in $M\left[G_{\lambda}\right][H]$. Since $k$ extends $j, \mu^{*}$ extends $\mu^{\prime}$. Also $\mu^{*}$ is in $M\left[G_{\nu}\right]$ since it is an element of $R(\nu)$.

We claim that in $M\left[G_{\nu}\right], \mu^{*}$ is a normal measure on $p_{\kappa} \nu^{\prime}$.

To see that $\mu^{*}$ is $\kappa$-additive let $\delta<\kappa$ and let $\left\langle A_{\alpha} ; \alpha<\delta\right\rangle$ be in $M\left[G_{\nu}\right]$ a sequence of sets so that $\mu^{*}\left(A_{\alpha}\right)=1$ for all $\alpha<\delta$. Set $B=\bigcap_{\alpha<\delta} A_{\alpha}$. Now $k(\delta)=j(\delta)=\delta$ since $\delta<\kappa$ and $j$ fixes every ordinal less than $k$. Hence $k(B)=$ $\bigcap_{\alpha<\delta} K\left(A_{\alpha}\right)$, and $j\left[\nu^{\prime}\right] \in k(B)$ because $j\left[\nu^{\prime}\right] \in k\left(A_{\alpha}\right)$ for all $\alpha<\delta$. So $\mu^{*}(B)$ $=1$.

Now fix $\alpha<\nu^{\prime}$. To see that if in $M\left[G_{\nu}\right], A=\left\{x \in P_{\kappa} \nu^{\prime}: \alpha \in x\right\}$ then $\mu^{*}(A)=1$, note that in $k\left(M\left[G_{\nu^{\prime}}\right]\right), k(A)=\left\{x \in p_{k(k)} k\left(\nu^{\prime}\right): k(\alpha) \in x\right\}$. But 
$\left|j\left[\nu^{\prime}\right]\right|=\nu^{\prime}$ and $k(\kappa)=j(\kappa)>\lambda^{\prime}>\nu^{\prime}$. Hence $j\left[\nu^{\prime}\right] \in k(A)$ and $\mu^{*}(A)=1$.

Finally we must show that in $M\left[G_{\nu}\right]$, if $f: p_{\kappa} \nu^{\prime} \rightarrow \nu^{\prime}$ is so that the set $A=$ $\left\{x \in p_{k} \nu^{\prime}: f(x) \in x\right\}$ has measure one with respect to $\mu^{*}$ then for some $\alpha<\nu^{\prime}$, $\mu^{*}\left(\left\{x \in p_{\kappa} \nu^{\prime}: f(x)=\alpha\right\}\right)=1$. Note that in $k\left(M\left[G_{\nu^{\prime}}\right]\right), k(A)=\left\{x \in p_{k(\kappa)} k\left(\nu^{\prime}\right):\right.$ $k(f)(x) \in x\}$, and that since $j\left[\nu^{\prime}\right] \in k(A), k(f)\left(j\left[\nu^{\prime}\right]\right) \in j\left[\nu^{\prime}\right]$. So there is an $\alpha \in \nu^{\prime}$ so that $k(f)\left(j\left[\nu^{\prime}\right]\right)=k \alpha$. It follows that in $M\left[G_{\nu}\right]$,

$$
\mu^{*}\left(\left\{x \in p_{\kappa} \nu^{\prime}: f(x)=\alpha\right\}\right)=1 .
$$

This concludes the proofs of the claim, lemma, and theorem.

The concept of ordinal definability is originally due to Gödel. Myhill and Scott rediscovered it, and we refer the reader to their paper [6] for a detailed exposition and relevant results.

The transitive closure of a set $x, \operatorname{Tc}(x)$, is the intersection of all transitive sets $A$ so that $x \in A . \quad x$ is ordinal definable if there is a formula $\phi\left(\nu_{0}, \ldots, \nu_{n+1}\right)$ of $\mathrm{ZF}$ and ordinals $\alpha_{0}<\cdots<\alpha_{n}<\beta$ so that $R(\beta) \vDash\left((\exists ! v) \phi\left(\alpha_{0}, \ldots, \alpha_{n}, v\right)\right.$ $\left.\wedge \phi\left(\alpha_{0}, \ldots, \alpha_{n}, x\right)\right) . x$ is hereditarily ordinal definable if every element of $\operatorname{Tc}(x)$ is ordinal definable. There is a sentence, $V=$ HOD, of ZF which asserts that every set is hereditarily ordinal definable.

The definable well-ordering of all pairs of ordinals due to Gödel gives rise to a term $\pi\left(v_{0}, v_{1}\right)$ of ZF so that for every cardinal $\nu, \pi \mid(\nu \times \nu)$ is a bijection from $\nu \times \nu$ onto $\nu$.

Suppose that $\nu$ is a Beth fixed point, $A$ is a subset of $\nu$, and $f$ is a bijection from $\nu$ onto $R(\nu)$ so that for every $(\beta, \alpha) \in \nu \times \nu, f(\beta) \in f(\alpha)$ iff $\pi(\beta, \alpha) \in A$. For such $A$ and $\nu$ we write " $A \sim R(\nu)$ ". An argument by induction on the wellfounded relation $S_{A}=\{(\beta, \alpha) \in \nu \times \nu: \pi(\beta, \alpha) \in A\}$ on $\nu$ establishes that the function $t_{A}$ from $\nu$ into $R(\nu)$ defined so that for every $\alpha \in \nu, t_{A}(\alpha)=\left\{t_{A}(\beta)\right.$ : $\pi(\beta, \alpha) \in A$ \} is precisely the function $f$. Then if $A$ is ordinal definable, $t_{A}$ and hence every element of $R(v)$ is ordinal definable.

If $\kappa$ is a supercompact cardinal then a class-sequence $\mu=\left\langle\mu_{\lambda}: \lambda\right.$ is a Beth fixed point $\geqslant k\rangle$ is a class-sequence of coherent measures for $k$ if for every pair of Beth fixed points $\lambda \geqslant \nu \geqslant k, \mu_{\lambda}$ is a normal measure on $p_{k} \lambda$ and $\mu_{\nu}$ is the projection of $\mu_{\lambda}$ on $p_{\kappa} \nu$, i.e., for every $A \subseteq p_{\kappa} \nu, \mu_{\nu}(A)=1$ iff $\mu_{\lambda}$ ( $\left\{x \in p_{\kappa} \lambda: x \cap\right.$ $\nu \in A\})=1$.

Suppose that $k$ is supercompact and that $\gamma>\kappa$ is an inaccessible cardinal. Let $\mu^{*}$ be a normal measure on $p_{k} \gamma$ and let $\mu=\left\{\left(\mu_{\lambda}, \lambda\right): \lambda\right.$ is a Beth fixed point in $[\kappa, \gamma)$ and $\mu_{\lambda}$ is the projection of $\mu^{*}$ on $\left.p_{\kappa} \lambda\right\}$. Then $A=\langle R(\gamma), R(\gamma+1)$; $\in\rangle$ is a model of Gödel-Bernays class-set theory, and in $A, \mu$ is a class-sequence of coherent measures for $k$.

Solovay has shown (unpublished) that if $k$ is supercompact with a class-sequence of coherent measures, then there are cardinals $\kappa^{\prime}<\lambda^{\prime}<\kappa$ so that $\lambda^{\prime}$ is 
inaccessible and $R\left(\lambda^{\prime}\right) \vDash \kappa^{\prime}$ is supercompact.

19. Lемма. Let $\mathrm{k}$ be a supercompact cardinal with a class sequence $\mu$ of coherent measures. For every Beth fixed point $\lambda \geqslant k$ let $j_{\lambda}: V \rightarrow j_{\lambda}(V) \simeq$ $V^{p_{k} \lambda} / \mu_{\lambda}$ be the elementary embedding of the universe associated with $\mu_{\lambda}$. There is a well-ordering $W$ of the universe so that for every Beth fixed point $\lambda \geqslant k$, the set $W_{\lambda}=W \cap R(\lambda)$ is a well-ordering of $R(\lambda)$ so that $j_{\lambda}\left(W_{\lambda}\right) \cap R(\lambda)=W_{\lambda}$, and so that $R(\lambda)$ is an initial segment with respect to $W$, that is, $(\forall x)(\forall y)(x \in$ $R(\lambda)$ and $y \notin R(\lambda) \rightarrow(x, y) \in W)$.

Proof. For the remainder of this proof let $\nu$ and $\lambda$ range over the Beth fixed points.

Select a well ordering $W_{\kappa}$ of $R(\kappa)$ so that for every $\nu<\kappa, R(\nu)$ is an initial segment of $R(k)$ with respect to $W_{k}$. For every $\lambda>_{k}$ let $W_{\lambda}=j_{\lambda}\left(W_{k}\right) \cap R(\lambda)$. Since $j_{\lambda}(V)$ is closed under $\lambda$-sequences, $W_{\lambda}$ is a well ordering of $R(\lambda)$ with the property that for every $\nu<\lambda, R(\nu)$ is an initial segment of $R(\lambda)$ with respect to $W_{\lambda}$. Since $(\forall x \in R(k))\left(x \in W_{\kappa} \leftrightarrow x \in W_{\lambda}\right), j_{\lambda}(V) \vDash\left(\forall x \in R\left(j_{\lambda}(k)\right)\right)$ $\left(x \in j_{\lambda}\left(W_{k}\right) \leftrightarrow x \in j_{\lambda}\left(W_{\lambda}\right)\right)$. Hence $j_{\lambda}\left(W_{\lambda}\right) \cap R(\lambda)=W_{\lambda}$.

Now fix $k<\nu<\lambda$. There is an elementary embedding $k: j_{\nu}(V) \rightarrow j_{\lambda}(V)$ so that $k \circ j_{\nu}=j_{\lambda}$ and so that for every $x \in R(\nu+1) k(x)=x$. Then $W_{\nu}=$ $k\left(W_{\nu}\right)=k\left(j_{\nu}\left(W_{k}\right) \cap R(\nu)\right)=j_{\lambda}\left(W_{k}\right) \cap R(\nu)=W_{\lambda} \cap R(\nu)$.

Set $W=\bigcup_{\lambda>k} W_{\lambda}$.

For the next two theorems let $M$ be a countable standard model of GödelBernays class-set theory so that $\boldsymbol{k}$ is a supercompact cardinal in $M$ and $\mu$ is a class sequence of $M$ of coherent measures for $\kappa$. We will work in $M$. " $\nu$ " and " $\lambda$ " and subscripted versions thereof will range over the Beth fixed points of $M$. For $\lambda>\kappa, \mu_{\lambda}, j_{\lambda}, W_{\lambda}$ and $W$ will be as in the preceding lemma.

20. THEOREM. There is a Cohen extension $N$ of $M$ so that $N$ is a model of "ZFC $+V=$ HOD" and $k$ is supercompact in $N$.

Proof. First a definition. Suppose that $\nu$ is a Beth fixed point and $A \subseteq$ $\nu$. Define the function $f$ on the set $\{\gamma:(\exists \alpha<\nu)(\gamma=\omega(\nu+\alpha+1))\}$ so that for every $\alpha<\nu, f(\omega(\nu+\alpha+1))=\omega(\nu+\alpha+3)$ if $\alpha \in A$ and $f(\omega(\nu+\alpha+1))$ $=\omega(\nu+\alpha+2)$ if $\alpha \notin A$. There is a term of ZF, $E^{*}\left(v_{0}, v_{1}\right)$, so that for $A, \nu$, and $f$ as above $E^{*}(\nu, A)=E(f)$ [cf. 16].

Work in $M$. Let $e$ be a class-function enumerating in increasing order the Beth fixed points.

Define by induction on the ordinals a very fine sequence of prosets $\left\langle P_{\alpha}\right.$; $\alpha$ an ordinal $>$ so that for every $\alpha$ and $\beta,\left|P_{\alpha}\right|<e(\alpha+2)$, and $e(\beta)^{\gamma}$ is a Beth fixed point in $M^{\left(B_{\alpha}\right)}$. 
Case I. For $\alpha=0$, let $P_{\alpha}=\langle\{0\} ;=\rangle$.

Case II. For $\alpha=\beta+1$ let $R$ be such that in $M^{\left(B_{\beta}\right)}, R=C\left(e(\beta)^{\gamma}, e(\alpha)^{\gamma}\right)$. Let $A$ be the $W$-least set so that in $M^{\left(B\left(P_{\beta} \widetilde{\otimes} R\right)\right)}, A \sim R\left(e(\beta)^{\vee}\right)$. Then define in $M^{\left(B\left(P_{\beta} \tilde{\otimes} R\right)\right)}, L=E^{*}\left(e(\beta)^{r}, A\right)$, and set $P_{\beta+1}=\left(P_{\beta} \widetilde{\otimes} R\right) \widetilde{\otimes} L$.

Case III. For $\alpha$ an inaccessible cardinal, let $P_{\alpha}$ be the direct limit of its predecessors.

Case IV. For $\alpha$ a limit ordinal that is not an inaccessible cardinal, let $P_{\alpha}$ be the inverse limit of its predecessors.

Let $P_{\infty}$ be the direct limit of the $P_{\alpha}$ 's and let $G_{\infty}$ be an M-generic subclass of $P_{\infty}$. Define $M\left[G_{\alpha}\right.$ ] and $M\left[G_{\infty}\right]=N$ as before [cf. 18 and remarks preceding 14]. By the closure properties of $E\left(v_{0}\right)$ and hence of $E^{*}\left(v_{0}, v_{1}\right)$, by 10 and 12 , and by $14, N$ is a model of ZFC.

For $\alpha=\beta+1, M\left[G_{\alpha}\right] \vDash$ (there is a subset $A$ of $e(\beta)$ so that $A \sim R(e(\beta)$ ) and so that for every $\gamma<e(\beta), \gamma \in A$ iff $2^{\omega(e(\beta)+\gamma+1)}=\omega(e(\beta)+\gamma+3)$. It follows that in $M\left[G_{\alpha}\right]$ every element of $R(e(\beta))$ is hereditarily ordinal definable. By the closure properties of $E\left(v_{0}\right)$ and by 10,12 , and $14, N \cap R(e(\alpha))=$ $M\left[G_{\alpha}\right] \cap R(e(\alpha))$. Hence in $N$ every element of $R(e(\beta))$ is hereditarily ordinal definable. More generally, $N \vDash(V=\mathrm{HOD})$.

One shows that $k$ is supercompact in $N$ by an argument analogous to that of the proof of Theorem 18. One uses the normal measure $\mu_{\lambda^{\prime}}$ on $p_{\kappa} \lambda^{\prime}$, the fact that $j_{\lambda^{\prime}}\left(W_{\lambda^{\prime}}\right) \cap R\left(\lambda^{\prime}\right)=W_{\lambda^{\prime}}$ and the following "local definability" property of the $P_{\alpha}$ 's which is analogous to Fact $B$ of 18 :

FACT B'. There is a term $v\left(v_{0}, v_{1}\right)$ of ZF so that if $\alpha$ is a fixed point of $e$ and $\beta\left\langle\alpha\right.$, then $v(\beta, W \cap R(\alpha))=P_{\beta}$ and $\langle R(\alpha) ; \in, W \cap R(\alpha)\rangle \vDash v(\beta, W \cap$ $R(\alpha))=P_{\beta}$.

Now suppose that in addition to the other properties of $M, M$ has a proper class of inaccessible cardinals.

21. THEOREM. There is a Cohen extension $N$ of $M$ so that $N$ is a model of "ZFC + G.C.H. $+V=$ HOD" and $k$ is supercompact in N.

Proof. For a coding device we use the term $\chi\left(v_{0}, v_{1}\right)$ of Lemma 17. This is the reason for the requirement that there be a proper class of inaccessibles in $M$. The proof is otherwise identical to the proof of Theorem 20 . We only describe the relevant class of forcing conditions.

Work in $M$. Let $e$ be a class-function enumerating in increasing order the closed unbounded class $\{\nu: \nu$ is a Beth fixed point so that $\mid\{\alpha<\nu: \alpha$ is inaccessible $\} \mid=\nu$.

Define by induction on the ordinals a very fine class-sequence of posets $\left\langle P_{\alpha} ; \alpha\right.$ an ordinal $\rangle$ so that for every $\alpha$ and $\beta,\left|P_{\alpha}\right|<e(\alpha+2)$, and $e(\beta)^{\gamma}$ is a Beth fixed point in $M^{\left(B_{\alpha}\right)}$. 
Case I. For $\alpha=0$, let $P_{\alpha}=\langle\{0\} ;=\rangle$.

Case II. For $\alpha=\beta+1$, define in $M^{\left(B_{\beta}\right)}, R=C\left(e(\beta)^{\gamma}, e(\alpha)^{\gamma}\right)$. Let $A$ be the $W$-least set so that $M^{\left(B\left(P_{\beta} \widetilde{\otimes} R\right)\right)} \vDash\left(A \sim R\left(e(\beta)^{2}\right)\right)$. In $M^{\left(B\left(P_{\beta} \widetilde{\otimes} R\right)\right)}$, let $L=$ $\chi\left(e(\beta)^{r}, A\right)$. Set $P_{\beta+1}=\left(P_{\beta} \widetilde{\otimes} R\right) \widetilde{\otimes} L$.

Case III. For $\alpha$ an inaccessible limit of inaccessibles, let $P_{\alpha}$ be the direct limit of its predecessors.

Case IV. For $\alpha$ a limit ordinal that is not an inaccessible limit of inaccessibles, let $P_{\alpha}$ be the inverse limit of its predecessors.

Theorems 20 and 21 translate by the usual methods to consistency results.

\section{BIBLIOGRAPHY}

1. W. B. Easton, Powers of regular cardinals, Ann. Math. Logic 1 (1970), 139-178. MR 42 \#392.

2. P. R. Halmos, Lectures on Boolean algebras, Van Nostrand Math. Studies, no. 1, Van Nostrand, Princeton, NJ., 1963. MR 29 \#4713.

3. T. J. Jech, Lectures in set theory, with particular emphasis on the method of forcing, Lecture Notes in Math., vol. 217, Springer-Verlag, Berlin and New York, 1971. MR 48 \#105.

4. - Trees, J. Symbolic Logic 36 (1971), 1-14. MR 44 \#1560.

5. M. Magidor, Dissertation, University of Jerusalem, 1972.

6. J. R. Myhill and D. Scott, Ordinal definability, Proc. Sympos. Pure Math., vol. 13, part 1, Amer. Math. Soc., Providence, R.I., 1971, pp. 271-278. MR 43 \#7318.

7. W. Reinhardt and R. Solovay, Strong axioms of infinity and elementary embeddings (to appear).

8. D. Scott and R. Solovay, Boolean-valued models for set theory, mimeographed notes.

9. J. R. Shoenfield, Unramified forcing, Proc. Sympos. Pure Math., vol. 13, part 1, Amer. Math. Soc., Providence, R.I., 1971, pp. 357-381. MR 43 \#6079.

10. J. Silver, The independence of Kurepa's conjecture and two-cardinal conjectures in model theory, Proc. Sympos. Pure Math., vol. 13, part 1, Amer. Math. Soc., Providence, R.I., 1971, pp. 383-390. MR 43 \#3112.

11. - Forthcoming paper on large cardinals and the G.C.H.

12. R. M. Solovay and S. Tennenbaum, Iterated Cohen extensions and Souslin's problem, Ann. of Math. (2) 94 (1971), 201-245. MR 45 \#3212.

13. D. H. Stewart, M. Sci. Thesis, Bristol, 1966.

14. F. Tall, Doctoral Dissertation, University of Wisconsin, 1969.

DEPARTMENT OF MATHEMATICS, UNIVERSITY OF CALIFORNIA, BERKELEY, CALIFORNIA 94720

DEPARTMENT OF MATHEMATICS, UNIVERSITY OF CALIFORNIA, LOS ANGELES, CALIFORNIA 90024 (Current address) 